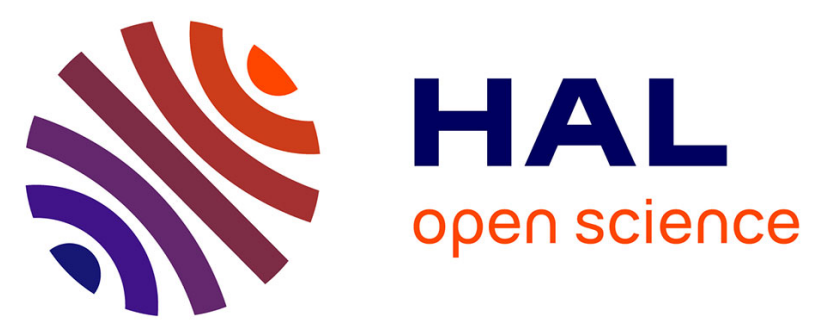

\title{
Nonparametric probabilistic approach of model uncertainties introduced by a projection-based nonlinear reduced-order model
}

Christian Soize, Charbel Farhat

\section{- To cite this version:}

Christian Soize, Charbel Farhat. Nonparametric probabilistic approach of model uncertainties introduced by a projection-based nonlinear reduced-order model . ECCOMAS 2016, 7th European Congress on Computational Methods in Applied Sciences and Engineering, Jun 2016, Island of Crete, Greece. pp.1-26. hal-01353199

\author{
HAL Id: hal-01353199 \\ https://hal.science/hal-01353199
}

Submitted on 10 Aug 2016

HAL is a multi-disciplinary open access archive for the deposit and dissemination of scientific research documents, whether they are published or not. The documents may come from teaching and research institutions in France or abroad, or from public or private research centers.
L'archive ouverte pluridisciplinaire $\mathbf{H A L}$, est destinée au dépôt et à la diffusion de documents scientifiques de niveau recherche, publiés ou non, émanant des établissements d'enseignement et de recherche français ou étrangers, des laboratoires publics ou privés. 


\title{
NONPARAMETRIC PROBABILISTIC APPROACH OF MODEL UNCERTAINTIES INTRODUCED BY A PROJECTION-BASED NONLINEAR REDUCED-ORDER MODEL
}

\author{
C. Soize ${ }^{1}$, C. Farhat $^{2}$ \\ ${ }^{1}$ Laboratoire Modélisation et Simulation Multi Echelle, MSME UMR 8208 CNRS, \\ Université Paris-Est, 5 bd Descartes, 77454 Marne-la-Vallee, France \\ e-mail: christian.soize@univ-paris-est.fr \\ ${ }^{2}$ Department of Aeronautics and Astronautics, Department of Mechanical Engineering, and Institute \\ for Computational and Mathematical Engineering, \\ Stanford University, Stanford, CA 94305, USA \\ e-mail: cfarhat@stanford.edu
}

Keywords: Modeling errors, Model uncertainties, Nonparametric probabilistic approach, Reducedorder model, Model order reduction, Uncertainty quantification.

\begin{abstract}
The paper is devoted to model uncertainties (or model form uncertainties) induced by modeling errors in computational sciences and engineering (such as in computational structural dynamics, fluid-structure interaction, and vibroacoustics, etc.) for which a parametric high-fidelity computational model (HFM) is used for addressing optimization problems (such as a robust design optimization problem), which are solved by introducing a parametric reducedorder model (ROM) constructed using an adapted reduced-order basis (ROB) derived from the parametric HFM. Two main methodologies are available to take into account such modeling errors. The first one is the usual output-predictive error method that has been introduced for many years. This approach can induce some difficulties because the parametric HFM and ROM do not learn from data. The second one is the nonparametric probabilistic approach of model uncertainties introduced in the framework of structural dynamics fifteen years ago. This approach is adapted, but is mainly limited to linear operators of the parametric HFM. The present paper deals with this challenging problem and proposes a novel nonparametric probabilistic approach of the modeling errors for any parametric nonlinear HFM for which a parametric nonlinear ROM can be constructed from the HFM. The methodology proposed consists in substituting the deterministic ROB with a stochastic ROB for which the probability measure in constructed on a subset of a compact Stiefel manifold. The stochastic model depends on a small number of hyperparameters for which the identification is performed by solving a statistical inverse problem. An application is presented in nonlinear computational structural dynamics.
\end{abstract}




\section{INTRODUCTION}

\subsection{Short overview on the high-dimensional and projection-based reduced-order models}

The potential of Partial Differential Equation (PDE)-based, High-Dimensional computational Models (HDMs) for enhancing system performance and predicting the unknown is recognized in almost every field of science and engineering. However, in many computational mechanics applications, nonlinear, time-dependent numerical simulations based on HDMs remain so cost-prohibitive that they cannot be used as often as needed. For this reason, nonlinear, projection-based Model Order Reduction (MOR) has recently emerged as a promising if not indispensable numerical tool for parametric applications such as, to name only a few, design, design optimization, statistical analysis, and simulation-based decision making [1, 2, 3, 4, 5, 6, 7, 8, 9, 10]. In general, a $\boldsymbol{\mu}$-parametric, high-fidelity, physics-based computational model is high-dimensional because its underlying spatial discretization is performed $a$ priori. On the other hand, the projection of such a computational model of dimension $N$ onto a subspace of low dimension $n \ll N$ represented by an associated $(N \times n)$ Reduced-Order Basis (ROB) $[V]$ leads to a Reduced-Order Model (ROM) of much lower dimension $n$. When $[V]$ is carefully constructed a posteriori, the corresponding ROM can capture the dominant behavior of the underlying $\boldsymbol{\mu}$-parametric HDM and therefore retain most of its fidelity. In general, knowledge about the system response is obtained during a training procedure that is performed offline. During this procedure, the model parameters represented here by the parameter vector $\boldsymbol{\mu}=\left(\mu_{1}, \ldots, \mu_{N_{p}}\right)$ belonging to the parameter space $\mathcal{C}_{\boldsymbol{\mu}}$ are sampled at a few points using a greedy but effective sampling strategy (for example, see [11]), and a set of problems related to the main problem of interest are solved to obtain a set of parametric solution snapshots. Then, these snapshots are compressed using, for example, the Singular Value Decomposition (SVD) to construct a global ROB. In general, the sampling strategy is designed so that the global ROB is reliable in a large region of the model parameter domain. Unfortunately, despite its low dimension, the resulting global (or $\boldsymbol{\mu}$-parametric) ROM does not necessarily guarantee computational feasibility. This is because the construction of this projection-based ROM scales not only with its size $n$, but also with that of the underlying HDM, $N \gg n$. In the deterministic setting, this issue is particularly problematic for nonlinear problems because the ROM needs to be repeatedly reconstructed to address, for example, time-dependency or Newton iterations for implicit solution strategies. This caveat is remedied by equipping a MOR method with a rigorous procedure for approximating the resulting reduced operators whose computational complexity scales only with the small size $n$ of the ROM [1, 3, 12, 13]. Such a procedure is also known in the literature as hyper reduction [14]. It transforms the nonlinear ROM into a hyper reduced ROM that guarantees feasibility, while maintaining as much as possible a desired level of accuracy. From these reasons, a nonlinear ROM or hyper reduced ROM inherits the modeling errors and associated uncertainties of its underlying HDM, including model form uncertainties. It is also tainted by additional errors introduced by the reduction processes highlighted above. Hence, if MOR is essential for enabling simulation-based decision making, Uncertainty Quantification (UQ) for ROMs is critical for certifying the decisions they enable.

\subsection{Parametric probabilistic approach of uncertainties using the $\mu$-parametric ROM}

The $\boldsymbol{\mu}$-parametric ROM has been used in the context of the parametric probabilistic approach of uncertainties, which consists in constructing prior and posterior stochastic models of the uncertain model-parameters (geometry, boundary conditions, material properties, etc) of the $\boldsymbol{\mu}$-parametric HFM and of the associated $\boldsymbol{\mu}$-parametric ROM (see for instance [15, 16, 17, 18, 
$19,20,21,22,23,24,25])$. Such an approach is very well adapted and very efficient to take into account the uncertainties in the $\boldsymbol{\mu}$-parametric HFM and/or in the $\boldsymbol{\mu}$-parametric ROM (see for instance [26, 4, 27]), and for large scale statistical inverse problems, see [28, 29, 30, 31, 32, 33, 34, 35]). However, the parametric probabilistic approach has not the capability to take into account model uncertainties induced by modeling errors that are introduced during the construction of the $\boldsymbol{\mu}$-parametric HFM and of the associated $\boldsymbol{\mu}$-parametric ROM [25, 27, 36].

\subsection{Nonparametric probabilistic approach of uncertainties using the $\mu$-parametric ROM}

The nonparametric probabilistic approach of model uncertainties induced by modeling errors has been introduced in 1999 [36, 37, 38, 39] for linear structural dynamic systems. It is organized around two steps. The first one consists in constructing a linear reduced-order model (ROM) of dimension $n$, from a linear HFM with $N$ degrees-of-freedom (dofs), by using an adapted ROB represented by the $(N \times n)$ matrix $[V]$. The second step consists in constructing a SROM by substituting the deterministic matrices of the ROM (such as the mass, the damping, and the stiffness reduced matrices) with random matrices for which the probability distributions are constructed using the Maximum Entropy (MaxEnt) principle [40, 41, 42] under the constraints defined by available information assocaited with algebraic properties (such as lower bounds, positiveness, integrability of the inverse, etc) and statistical information (such as the mean value equals to the nominal values, etc), and for which advanced algorithms have been developed for the high dimensions [39, 43, 44]. This approach has been extended to different ensembles of random matrices (see [38, 45]), static boundary value problems [46], and has been experimentally validated and applied in many areas, including: dynamics of composite structures [47] and viscoelastic structures [48, 49], dynamic substructuring techniques [50, 51, 52, 53, 54], vibroacoustic systems [48, 55, 56, 57], soil-structure interactions and earthquake engineering [58, 59, 60], robust design and optimization [61, 62], to name only a few. More recently, this nonparametric probabilistic approach has been extended in structural dynamics to nonlinear geometrical effects [63, 64]. Such an extension is very efficient but is strongly related to the mathematical properties of the nonlinear elasticity operator. Such an extension cannot, a priori, be carried out for any nonlinear operator and the nonparametric probabilistic approach of model uncertainties induced by modeling errors has not received yet a solution for general nonlinear dynamical systems in computational solid mechanics and computational fluid dynamics. Hence, the objective of this paper is to propose a nonparametric probabilistic approach for such nonlinear dynamical systems, for which the framework is detailed below.

\subsection{Model uncertainties induced by the use of a $\mu$-independent ROB for constructing the $\mu$-parametric ROM}

As explained at the end of Section 1.1, despite all the mathematical analyses and the advanced methodologies that have been developed, and despite all the precautions taken to build a $\boldsymbol{\mu}$-parametric nonlinear ROM from a $\boldsymbol{\mu}$-parametric nonlinear HFM, the efficiency of the $\boldsymbol{\mu}$ parametric nonlinear ROM depends on the sampling points $\boldsymbol{\mu}_{1}, \ldots, \boldsymbol{\mu}_{m_{\mu}}$ chosen for $\boldsymbol{\mu}$ in $\mathcal{C}_{\boldsymbol{\mu}}$ for constructing the ROB, $[V]$, and depends on the accuracy of the constructed approximation. In other words, it depends of the dimension $n$ chosen for the $\boldsymbol{\mu}$-parametric ROM. Even if the $\boldsymbol{\mu}$ parametric nonlinear ROM that is constructed has the capability to give a good approximation of the $\boldsymbol{\mu}$-parametric nonlinear HFM, the CPU-time consideration leads to limiting the size $m_{\mu}$ of the sampling points for $\boldsymbol{\mu}$ and the dimension $n$ of the ROB, which induces an error between the 
$\boldsymbol{\mu}$-parametric nonlinear ROM and the $\boldsymbol{\mu}$-parametric nonlinear HFM when $\boldsymbol{\mu}$ runs through $\mathcal{C}_{\boldsymbol{\mu}}$.

\subsection{Objective and organization of the paper}

To this effect, the objective of this paper is to propose a nonparametric probabilistic approach for model uncertainties induced by two types of modeling errors:

- The first type of modeling errors is associated with the usage, for the purpose of computational efficiency, of the $\boldsymbol{\mu}$-parametric nonlinear ROM as a surrogate of the $\boldsymbol{\mu}$-parametric nonlinear HFM. The dimension $m_{\boldsymbol{\mu}}$ of the parameter $\boldsymbol{\mu}$ can be small or large. When the dimension $n$ of the $\boldsymbol{\mu}$-parametric nonlinear ROM goes to $N$ and the number $m_{\mu}$ of the sampling points of $\boldsymbol{\mu}$ in $\mathcal{C}_{\boldsymbol{\mu}}$ (used for constructing $[V]$ ) goes to infinity, the modeling error between the $\boldsymbol{\mu}$-parametric nonlinear ROM and the $\boldsymbol{\mu}$-parametric nonlinear HFM goes to zero.

- The second type of modeling errors is due to the modeling errors introduced in the construction of the $\boldsymbol{\mu}$-parametric nonlinear HFM itself. This means that the HFM does not perfectly predict the experimental data for the quantities of interest.

Consequently, the distance between the predictions of the $\boldsymbol{\mu}$-parametric ROM and the experimental data are due to the two types of modeling errors.

In this paper, we give a short presentation of a novel nonparametric probabilistic approach that is detailed in [65]. Section 2 deals with a presentation of the nonparametric probabilistic approach for model uncertainties induced by the modeling errors in the nonlinear reduced-order models. Section 3 is devoted to the probabilistic construction of the stochastic reduced-order basis (SROB). The theory is presented in a discrete form that can be immediately exploited by computational models. Section 4 is devoted to a simple application in nonlinear computational structural dynamics that is easy to reproduce by the interested reader.

\section{NONPARAMETRIC PROBABILISTIC APPROACH FOR UNCERTAINTY ANAL- YSIS OF NONLINEAR MODEL ORDER REDUCTION METHODS}

An example of a $\boldsymbol{\mu}$-parametric nonlinear HFM is chosen in order to explain what is the nonparametric probabilistic approach proposed for taking into account the two types of modeling errors:

$\diamond$ the errors induced by the use of the $\boldsymbol{\mu}$-parametric nonlinear ROM instead of the $\boldsymbol{\mu}$-parametric nonlinear HFM,

$\diamond$ the modeling errors introduced during the construction of the $\boldsymbol{\mu}$-parametric nonlinear HFM.

\subsection{Description of the $\mu$-parametric nonlinear HFM in the field of nonlinear structural dynamics}

We consider a $\boldsymbol{\mu}$-parametric nonlinear computational dynamical model on $\mathbb{R}^{N}$ corresponding to the finite element discretization of a structure,

$$
\left.[M] \ddot{\mathbf{y}}(t)+\mathbf{g}(\mathbf{y}(t), \dot{\mathbf{y}}(t) ; \boldsymbol{\mu})=\mathbf{f}(t ; \boldsymbol{\mu}) \quad, \quad t \in] t_{0}, T\right],
$$

with the initial conditions

$$
\mathbf{y}\left(t_{0}\right)=\mathbf{y}_{0} \quad, \quad \dot{\mathbf{y}}\left(t_{0}\right)=\mathbf{y}_{1}
$$


and with $N_{\text {cD }}<N$ constraint equations that are written in a matrix form as

$$
[B]^{T} \mathbf{y}(t)=\mathbf{0}_{N_{\mathrm{CD}}} \quad, \quad t \in\left[t_{0}, T\right],
$$

in which $t_{0}$ and $T$ are given such that $-\infty<t_{0}<T<+\infty$, where $\mathbf{y}_{0}$ and $\mathbf{y}_{1}$ are two given vectors in $\mathbb{R}^{N}$ satisfying the constraint equation. In Eq. (1), $\boldsymbol{\mu}$ is the parameter that belongs to $\mathcal{C}_{\boldsymbol{\mu}} \subset \mathbb{R}^{m_{\boldsymbol{\mu}}}$ introduced before, $t$ denotes the time variable, $\mathbf{y}(t)=\left(y_{1}(t), \ldots, y_{N}(t)\right)$ is the $\mathbb{R}^{N}$ vector of the $N$ dofs, $\dot{\mathbf{y}}(t)=d \mathbf{y}(t) / d t$ and $\ddot{\mathbf{y}}(t)=d^{2} \mathbf{y}(t) / d t^{2}$ are the velocity and the acceleration vectors, $[M]$ is the mass matrix that belongs to $\mathbb{M}_{N}^{+}$and which is assumed to be independent of $t$ and $\boldsymbol{\mu}$, where $\mathbf{g}(\mathbf{y}(t), \dot{\mathbf{y}}(t) ; \boldsymbol{\mu})$ is the $\mathbb{R}^{N}$ vector representing the internal forces at time $t$, which depends on $\mathbf{y}(t), \dot{\mathbf{y}}(t)$, and $\boldsymbol{\mu}$, and finally, where $\mathbf{f}(t ; \boldsymbol{\mu})$ is the $\mathbb{R}^{N}$ vector of the external forces at time $t$, which depends on parameter $\boldsymbol{\mu}$. In Eq. (3), $[B]$ is a given matrix in $\mathbb{M}_{N, N_{\mathrm{CD}}}$, which defines the $N_{\mathrm{CD}}$ constraints on $\mathbf{y}$, and which is assumed to be independent of $t$ and $\boldsymbol{\mu}$ and such that $[B]^{T}[B]=\left[I_{N_{\mathrm{CD}}}\right]$. The $\mathbb{R}^{N}$-valued solution $\left\{\mathbf{y}(t ; \boldsymbol{\mu}), t \in\left[t_{0}, T\right]\right\}$ of Eqs. (1) to (3) depends on $\boldsymbol{\mu}$. At time $t$, the quantity of interest (QoI) is a vector $\mathbf{o}(t ; \boldsymbol{\mu})=$ $\left(o_{1}(t ; \boldsymbol{\mu}), \ldots, o_{m_{\mathbf{0}}}(t ; \boldsymbol{\mu})\right)$ with values in $\mathbb{R}^{m_{\mathbf{0}}}$, depending on $\boldsymbol{\mu}$, which is written as

$$
\mathbf{o}(t ; \boldsymbol{\mu})=\mathbf{h}(\mathbf{y}(t ; \boldsymbol{\mu}), \dot{\mathbf{y}}(t ; \boldsymbol{\mu}), \mathbf{f}(t ; \boldsymbol{\mu}), t ; \boldsymbol{\mu}) \quad, \quad t \in\left[t_{0}, T\right],
$$

in which $\mathbf{h}$ is a given mapping.

\subsection{Construction of a $\mu$-parametric nonlinear ROM associated with the $\mu$-parametric nonlinear HFM}

Let $[V] \in \mathbb{M}_{N, n}$ be a ROB independent of $\boldsymbol{\mu}$, where $n \ll N$ is the dimension of the ROB that is assumed to be constructed for representing the solution $\left\{\mathbf{y}(t ; \boldsymbol{\mu}), t \in\left[t_{0}, T\right]\right\}$ for all $\boldsymbol{\mu} \in \mathcal{C}_{\boldsymbol{\mu}}$ (as explained before), and satisfies $[V]^{T}[M][V]=\left[I_{n}\right]$. By construction, this ROB satisfies also the constraint equation $[B]^{T}[V]=\left[0_{N_{\mathrm{cD}}, n}\right]$. It is assumed that the $\boldsymbol{\mu}$-parametric nonlinear ROM is constructed via the Galerkin method which yields

$$
\begin{gathered}
\mathbf{y}^{(n)}(t)=[V] \mathbf{q}(t) \quad, \quad t \in\left[t_{0}, T\right], \\
\left.\left.\ddot{\mathbf{q}}(t)+[V]^{T} \mathbf{g}([V] \mathbf{q}(t),[V] \dot{\mathbf{q}}(t) ; \boldsymbol{\mu})=[V]^{T} \mathbf{f}(t ; \boldsymbol{\mu}) \quad, \quad t \in\right] t_{0}, T\right],
\end{gathered}
$$

with the initial conditions

$$
\mathbf{q}\left(t_{0}\right)=[V]^{T}[M] \mathbf{y}_{0} \quad, \quad \dot{\mathbf{q}}\left(t_{0}\right)=[V]^{T}[M] \mathbf{y}_{1} .
$$

The $\mathbb{R}^{n}$-valued solution $\left\{\mathbf{q}(t ; \boldsymbol{\mu}), t \in\left[t_{0}, T\right]\right\}$ of Eqs. (6) and (7) depends on $\boldsymbol{\mu}$, and $\left\{\mathbf{y}^{(n)}(t ; \boldsymbol{\mu})\right.$, $\left.t \in\left[t_{0}, T\right]\right\}$ is the $n$-order approximation of $\left\{\mathbf{y}(t ; \boldsymbol{\mu}), t \in\left[t_{0}, T\right]\right\}$. The corresponding approximation $\mathbf{0}^{(n)}$ of $\mathbf{o}$ is given by

$$
\mathbf{o}^{(n)}(t ; \boldsymbol{\mu})=\mathbf{h}\left(\mathbf{y}^{(n)}(t ; \boldsymbol{\mu}), \dot{\mathbf{y}}^{(n)}(t ; \boldsymbol{\mu}), \mathbf{f}(t ; \boldsymbol{\mu}), t ; \boldsymbol{\mu}\right) \quad, \quad t \in\left[t_{0}, T\right] \quad, \quad \boldsymbol{\mu} \in \mathcal{C}_{\boldsymbol{\mu}}
$$

For a given reduced-order $n$, the error induced by the use of the $\boldsymbol{\mu}$-parametric nonlinear ROM instead of the $\boldsymbol{\mu}$-parametric nonlinear HFM could, a priori, be estimated (in the $L^{2}$ sense) by

$$
\varepsilon(n)=\int_{\mathcal{C}_{\mu}} \int_{t_{0}}^{T}\left\|\mathbf{o}(t ; \boldsymbol{\mu})-\mathbf{o}^{(n)}(t ; \boldsymbol{\mu})\right\|^{2} d t d \boldsymbol{\mu} .
$$


It should be noted that the estimation of $\varepsilon(n)$ defined by Eq. (9) must require the computation of the solution of the HFM for a very large number of sampled values of the vector-valued parameter $\boldsymbol{\mu}$ in $\mathcal{C}_{\boldsymbol{\mu}}$ in order to correctly estimate the integral in $\boldsymbol{\mu}$ over $\mathcal{C}_{\boldsymbol{\mu}}$. In practice, such a computation cannot be done and Eq. (9) must be replaced by

$$
\varepsilon(n)=\sum_{i=1}^{m_{\mu}} \int_{t_{0}}^{T}\left\|\mathbf{o}\left(t ; \boldsymbol{\mu}_{i}\right)-\mathbf{o}^{(n)}\left(t ; \boldsymbol{\mu}_{i}\right)\right\|^{2} d t,
$$

in which $\boldsymbol{\mu}_{1}, \ldots, \boldsymbol{\mu}_{m_{\mu}}$ are the sampling points used for constructing the ROB $[V]$.

\subsection{Construction of a stochastic ROB (SROB) with the proposed nonparametric proba- bilistic approach}

The proposed nonparametric probabilistic approach of model uncertainties induced by modeling errors consists in substituting the deterministic ROB $[V]$ with a stochastic ROB $[\mathbf{W}]$ that is independent of $\boldsymbol{\mu}$, which is a random matrix with values in $\mathbb{M}_{N, n}$ for which the support of its probability distribution is the subset of $\mathbb{M}_{N, n}$ corresponding to the constraints $[\mathbf{W}]^{T}[M][\mathbf{W}]=$ $\left[I_{n}\right]$ and $[B]^{T}[\mathbf{W}]=\left[0_{N_{\mathrm{cD}}, n}\right]$ almost surely. The probability distribution of the random matrix $[\mathbf{W}]$ depends on a vector-valued hyperparameter $\boldsymbol{\alpha}=\left(\alpha_{1}, \ldots, \alpha_{m_{\boldsymbol{\alpha}}}\right)$ belonging to a subset $\mathcal{C}_{\boldsymbol{\alpha}}$ of $\mathbb{R}^{m_{\boldsymbol{\alpha}}}$ where the dimension $m_{\boldsymbol{\alpha}}$ will be chosen small in order for the statistical inverse problem for identifying $\boldsymbol{\alpha}$ to be feasible. The construction of the SROB is presented in Section 3 .

\subsection{Construction of the $\mu$-parametric nonlinear SROM associated with the $\mu$-parametric nonlinear ROM using the proposed nonparametric probabilistic approach}

The $\boldsymbol{\mu}$-parametric nonlinear SROM associated with the $\boldsymbol{\mu}$-parametric nonlinear ROM is deduced from Eqs. (5) to (8) by replacing $[V]$ with the random matrix $[\mathbf{W}]$. Consequently, $\mathbf{y}^{(n)}, \mathbf{q}$, and $\mathbf{o}^{(n)}$ become the stochastic processes $\mathbf{Y}^{(n)}, \mathbf{Q}$, and $\mathbf{O}^{(n)}$, and the SROM is written as

$$
\begin{gathered}
\mathbf{Y}^{(n)}(t)=[\mathbf{W}] \mathbf{Q}(t) \quad, \quad t \in\left[t_{0}, T\right], \\
\left.\left.\ddot{\mathbf{Q}}(t)+[\mathbf{W}]^{T} \mathbf{g}([\mathbf{W}] \mathbf{Q}(t),[\mathbf{W}] \dot{\mathbf{Q}}(t) ; \boldsymbol{\mu})=[\mathbf{W}]^{T} \mathbf{f}(t ; \boldsymbol{\mu}) \quad, \quad t \in\right] t_{0}, T\right],
\end{gathered}
$$

with the initial conditions

$$
\mathbf{Q}\left(t_{0}\right)=[\mathbf{W}]^{T}[M] \mathbf{y}_{0} \quad, \quad \dot{\mathbf{Q}}\left(t_{0}\right)=[\mathbf{W}]^{T}[M] \mathbf{y}_{1} .
$$

The $\mathbb{R}^{n}$-valued stochastic solution $\left\{\mathbf{Q}(t ; \boldsymbol{\mu}, \boldsymbol{\alpha}), t \in\left[t_{0}, T\right]\right\}$ of Eqs. (12) and (13) depends on $\boldsymbol{\mu} \in \mathcal{C}_{\boldsymbol{\mu}}$ and $\boldsymbol{\alpha} \in \mathcal{C}_{\boldsymbol{\alpha}}$. The stochastic process $\left\{\mathbf{Y}^{(n)}(t ; \boldsymbol{\mu}, \boldsymbol{\alpha}), t \in\left[t_{0}, T\right]\right\}$ is the $n$-order approximation of stochastic process $\left\{\mathbf{Y}(t ; \boldsymbol{\mu}), t \in\left[t_{0}, T\right]\right\}$. The corresponding approximation $\left\{\mathbf{O}^{(n)}(t ; \boldsymbol{\mu}, \boldsymbol{\alpha}), t \in\left[t_{0}, T\right]\right\}$ of the random quantity of interest $\left\{\mathbf{O}(t ; \boldsymbol{\mu}), t \in\left[t_{0}, T\right]\right\}$ is given, for all $t \in\left[t_{0}, T\right], \boldsymbol{\mu} \in \mathcal{C}_{\boldsymbol{\mu}}$, and $\boldsymbol{\alpha} \in \mathcal{C}_{\boldsymbol{\alpha}}$, by

$$
\mathbf{O}^{(n)}(t ; \boldsymbol{\mu}, \boldsymbol{\alpha})=\mathbf{h}\left(\mathbf{Y}^{(n)}(t ; \boldsymbol{\mu}, \boldsymbol{\alpha}), \dot{\mathbf{Y}}^{(n)}(t ; \boldsymbol{\mu}, \boldsymbol{\alpha}), \mathbf{f}(t ; \boldsymbol{\mu}), t ; \boldsymbol{\mu}\right) .
$$

\subsection{Identification of hyperparameter $\alpha$ of the probability distribution of $[\mathbf{W}]$}

The identification of the hyperparameter $\boldsymbol{\alpha} \in \mathcal{C}_{\boldsymbol{\alpha}} \subset \mathbb{R}^{m_{\alpha}}$ can be performed using the maximum likelihood method or a nonlinear least-squares method for the QoI. For instance, a nonlinear least-squares method can be formulated as follows for the two types of modeling errors. Let $J(\boldsymbol{\alpha})$ be the cost function defined on $\mathcal{C}_{\boldsymbol{\alpha}}$ by

$$
J(\boldsymbol{\alpha})=w_{J} J_{\text {mean }}(\boldsymbol{\alpha})+\left(1-w_{J}\right) J_{\text {std }}(\boldsymbol{\alpha}),
$$


in which $w_{J}$ is a weight such that $0 \leq w_{J} \leq 1$, and where $J_{\text {mean }}(\boldsymbol{\alpha})$ and $J_{\text {std }}(\boldsymbol{\alpha})$ allow for controlling the identification of $\boldsymbol{\alpha}$ with respect to the mean value and the statistical fluctuations,

$$
\begin{gathered}
J_{\text {mean }}(\boldsymbol{\alpha})=\frac{1}{c_{\text {mean }}\left(\boldsymbol{\mu}_{1}, \ldots, \boldsymbol{\mu}_{m_{\mu}}\right)} \sum_{i=1}^{m_{\mu}} \int_{t_{0}}^{T}\left\|\mathbf{o}^{\text {ref }}\left(t ; \boldsymbol{\mu}_{i}\right)-E\left\{\mathbf{O}^{(n)}\left(t ; \boldsymbol{\mu}_{i}, \boldsymbol{\alpha}\right)\right\}\right\|^{2} d t, \\
J_{\text {std }}(\boldsymbol{\alpha})=\frac{1}{c_{\text {std }}\left(\boldsymbol{\mu}_{1}, \ldots, \boldsymbol{\mu}_{m_{\mu}}\right)} \sum_{i=1}^{m_{\mu}} \int_{t_{0}}^{T}\left\|\mathbf{v}^{(\mathrm{ref}, n)}\left(t ; \boldsymbol{\mu}_{i}\right)-\mathbf{v}^{(n)}\left(t ; \boldsymbol{\mu}_{i}, \boldsymbol{\alpha}\right)\right\|^{2} d t .
\end{gathered}
$$

The positive constants $c_{\text {mean }}\left(\boldsymbol{\mu}_{1}, \ldots, \boldsymbol{\mu}_{m_{\mu}}\right)$ and $c_{\text {std }}\left(\boldsymbol{\mu}_{1}, \ldots, \boldsymbol{\mu}_{m_{\mu}}\right)$ are written as

$$
\begin{gathered}
c_{\text {mean }}\left(\boldsymbol{\mu}_{1}, \ldots, \boldsymbol{\mu}_{m_{\mu}}\right)=\sum_{i=1}^{m_{\mu}} \int_{t_{0}}^{T}\left\|\mathbf{o}^{\mathrm{ref}}\left(t ; \boldsymbol{\mu}_{i}\right)\right\|^{2} d t, \\
c_{\text {std }}\left(\boldsymbol{\mu}_{1}, \ldots, \boldsymbol{\mu}_{m_{\mu}}\right)=\sum_{i=1}^{m_{\mu}} \int_{t_{0}}^{T}\left\|\mathbf{v}^{(\mathrm{ref}, n)}\left(t ; \boldsymbol{\mu}_{i}\right)\right\|^{2} d t,
\end{gathered}
$$

where $\mathbf{v}^{(\mathrm{ref}, n)}\left(t ; \boldsymbol{\mu}_{i}\right)=\left(v_{1}^{(\mathrm{ref}, n)}\left(t ; \boldsymbol{\mu}_{i}\right), \ldots, v_{m_{\mathbf{0}}}^{(\mathrm{ref}, n)}\left(t ; \boldsymbol{\mu}_{i}\right)\right)$ is such that,

$$
v_{j}^{(\text {ref }, n)}\left(t ; \boldsymbol{\mu}_{i}\right)=\gamma\left|o_{j}^{\text {ref }}\left(t ; \boldsymbol{\mu}_{i}\right)-o_{j}^{(n)}\left(t ; \boldsymbol{\mu}_{i}\right)\right| \quad, \quad j=1, \ldots m_{\mathbf{o}},
$$

and where $\gamma>0$ allows for controlling the amplitude of the target related to the statistical fluctuations. In Eq. (17), $\mathbf{v}^{(n)}\left(t ; \boldsymbol{\mu}_{i}, \boldsymbol{\alpha}\right)=\left(v_{1}^{(n)}\left(t ; \boldsymbol{\mu}_{i}, \boldsymbol{\alpha}\right), \ldots, v_{m_{\mathbf{o}}}^{(n)}\left(t ; \boldsymbol{\mu}_{i}, \boldsymbol{\alpha}\right)\right)$ is such that

$$
\left.v_{j}^{(n)}\left(t ; \boldsymbol{\mu}_{i}, \boldsymbol{\alpha}\right)=\left\{E\left\{O_{j}^{(n)}\left(t ; \boldsymbol{\mu}_{i}, \boldsymbol{\alpha}\right)^{2}\right\}-\left(E\left\{O_{j}^{(n)}\left(t ; \boldsymbol{\mu}_{i}, \boldsymbol{\alpha}\right)\right\}\right)^{2}\right\}\right\}^{1 / 2}, \quad j=1, \ldots m_{\mathbf{o}} .
$$

In Eqs. (16), (18) and (20), $\mathbf{o}^{\text {ref }}$ is defined as a function of the type of the modeling errors that are taken into account:

$\diamond$ If only the errors induced by the use of the $\mu$-parametric nonlinear ROM instead of the $\boldsymbol{\mu}$-parametric nonlinear HFM are taken into account, then $\mathbf{o}^{\text {ref }}=\mathbf{0}$.

$\diamond$ If the two types of modeling errors are simultaneously taken into account (the modeling errors induced by the use of the $\boldsymbol{\mu}$-parametric nonlinear ROM instead of the $\boldsymbol{\mu}$-parametric nonlinear HFM and the modeling errors introduced during the construction of the $\mu$-parametric nonlinear HFM), then $\mathbf{0}^{\text {ref }}=\mathbf{o}^{\text {exp }}$ in which $\mathbf{o}^{\text {exp }}$ corresponds to experimental data for the QoI.

With such a formulation, in the cost function,

$\diamond \mathbf{0}^{\text {ref }}$ appears as the target for the mean value with a weight $w_{J}$,

$\diamond \mathbf{v}^{\text {(ref, } n)}$ appears as the target for the standard deviation with a weight $1-w_{J}$.

The identification of hyperparameter $\boldsymbol{\alpha}$ consists in calculating $\boldsymbol{\alpha}^{\text {opt }}$ such that

$$
\boldsymbol{\alpha}^{\mathrm{opt}}=\min _{\boldsymbol{\alpha} \in \mathcal{C}_{\boldsymbol{\alpha}}} J(\boldsymbol{\alpha})
$$

\subsection{Justification}

Why a random basis of the admissible set would be useful? Such a question is fully licit because it is well known that, with the Galerkin method, the solution $\mathbf{y}^{(n)}$ constructed using the $\mathrm{ROM}$ for an order $n$ for which $\mathbf{y}^{(n)}$ is close to $\mathbf{y}$ (convergence of $\mathbf{y}^{(n)}$ towards the solution $\mathbf{y}$ of 
the HFM when $n$ goes to $N$ ), is independent of the choice of the vector basis of the admissible set (such a statement is perfectly true for $n=N$ ). For a similar reason, for any SROB of the admissible set, the stochastic solution $\mathbf{Y}^{(n)}$ constructed using the SROM converges almost surely towards the deterministic solution $\mathbf{y}$ of the HFM, which is thus, at convergence, independent of the choice of the stochastic basis. However, and as we have previously explained, for $n \ll N$, and taking into account that the ROB $[V]$ is constructed for $\boldsymbol{\mu}_{1}, \ldots, \boldsymbol{\mu}_{m_{\mu}}$ in $\mathcal{C}_{\boldsymbol{\mu}}$, for all $\boldsymbol{\mu}$ fixed in $\mathcal{C}_{\boldsymbol{\mu}}$, the solution $\mathbf{y}^{(n)}$ of the ROM is only an approximation of $\mathbf{y}$, and consequently, there is an error introduced by the use of the ROM with order $n$ (the existence of such an error is the main assumption of the method that is proposed). In such a case, for a fixed value of $n$ for which the convergence is not reached, the value of the error depends on the choice of the basis of the admissible set (if the $n$ vectors of the basis are extracted from another family that constitutes another vector basis of the admissible set, then the solution $\mathbf{y}^{(n)}$ of the ROM is modified). Consequently, when the basis is substituted by the SROB $[\mathbf{W}]$, a stochastic family $\mathbf{Y}^{(n)}$ is generated using the SROM. The idea is then to adapt the statistical fluctuations of $\mathbf{Y}^{(n)}$ for representing the error between the ROM and the HFM, and also, as it has been explained before, to represent the modeling errors introduced in the construction of the $\boldsymbol{\mu}$-parametric nonlinear HFM itself.

\section{CONSTRUCTION OF A STOCHASTIC REDUCED-ORDER BASIS}

The construction of the SROM is detailed in [65]. Only the main ideas of the construction are given below. The ROB corresponds to a compact Stiefel manifold denoted by $\mathbb{S}_{N, n}$. Since the nonparametric probabilistic approach consists in defining the SROB by a probability measure on $\mathbb{S}_{N, n}$, we have to construct a parameterization of $\mathbb{S}_{N, n}$, which must be efficient for the high dimensions.

\subsection{Construction of a parameterization of the compact Stiefel manifold $\mathbb{S}_{N, n}$}

Let $[M] \in \mathbb{M}_{N}^{+}$be a given positive-definite symmetric $(N \times N)$ real matrix (possibly, $[M]$ can be $\left.\left[I_{N}\right]\right)$. The set $\mathbb{M}_{N, n}$ of all the $(N \times n)$ real matrices is considered as an Euclidean space equipped with the inner product $\ll\left[V_{1}\right],\left[V_{2}\right] \gg_{M}=\operatorname{tr}\left\{\left[V_{1}\right]^{T}[M]\left[V_{2}\right]\right\}$ and the associated norm $\|V\|_{M}=\left\{\operatorname{tr}\left\{[V]^{T}[M][V]\right\}\right\}^{1 / 2}$. Let $[V]$ be a reduced-order basis (ROB) belonging to the compact Stiefel manifold $\mathbb{S}_{N, n}$ defined (see for instance [66]) by

$$
\mathbb{S}_{N, n}=\left\{[V] \in \mathbb{M}_{N, n},[V]^{T}[M][V]=\left[I_{n}\right]\right\} \subset \mathbb{M}_{N, n},
$$

for which the dimension is $\nu_{\mathbb{S}}=N n-n(n+1) / 2=n(n-1) / 2+n(N-n)$. The tangent vector space, $T_{V} \mathbb{S}_{N, n}$, to $\mathbb{S}_{N, n}$ at point $[V] \in \mathbb{S}_{N, n}$ is defined by

$$
T_{V} \mathbb{S}_{N, n}=\left\{[Z] \in \mathbb{M}_{N, n}:[V]^{T}[M][Z]+[Z]^{T}[M][V]=\left[0_{n, n}\right]\right\} .
$$

The dimension of $T_{V} \mathbb{S}_{N, n}$ is $\nu_{\mathbb{S}}=n(n-1) / 2+n(N-n)$. We are interested in constructing a non classical parameterization of $\mathbb{S}_{N, n}$, which does not require the construction of a big matrix in $\mathbb{M}_{N, N-n}$, and which consists in using the projection $[Z]=\operatorname{Proj}_{T_{V} \mathbb{S}_{N, n}}([A])$ of any matrix $[A] \in \mathbb{M}_{N, n}$ onto $T_{V} \mathbb{S}_{N, n}$,

$$
[Z]=\operatorname{Proj}_{T_{V} \mathbb{S}_{N, n}}([A]):=[A]-[V][D] \quad, \quad[D]=\left([V]^{T}[M][A]+[A]^{T}[M][V]\right) / 2,
$$

in which $[D] \in \mathbb{M}_{n}^{S}$ is a $(n \times n)$ symmetric matrix.

Let $[Z] \mapsto R_{s, V}([Z])$ be the smooth mapping from tangent vector space $T_{V} \mathbb{S}_{N, n}$ of $\mathbb{S}_{N, n}$ at a given point $[V]$ into $\mathbb{S}_{N, n}$,

$$
[W]=R_{s, V}([Z])
$$


which is constructed in order that

$$
[V]=R_{s, V}\left(\left[0_{N, n}\right]\right)
$$

Consequently, for any $[Z]$ in $T_{V} \mathbb{S}_{N, n},[W]=R_{s, V}([Z])$ is such that $[W]^{T}[M][W]=\left[I_{n}\right]$. The construction of $R_{s, V}$ is performed by using the polar decomposition (see for instance [67]), that is adapted to Eq. (23). For any $[V]$ fixed in $\mathbb{S}_{N, n}$, mapping $R_{s, V}$ is written as

$$
[W]=R_{s, V}([Z]):=([V]+s[Z])\left[H_{s}(Z)\right] \quad, \quad[Z] \in T_{V} \mathbb{S}_{N, n},
$$

in which $s \geq 0$ is a real number $s \geq 0$ that is used for controlling the level of fluctuations of $[Z]$ in $T_{V} \mathbb{S}_{N, n}$ around $[V]$ in $\mathbb{S}_{N, n}$, and where the positive-definite matrix $\left[H_{s}(Z)\right]$ in $\mathbb{M}_{n}^{+}$is defined by

$$
\left[H_{s}(Z)\right]=\left(\left[I_{n}\right]+s^{2}[Z]^{T}[M][Z]\right)^{-1 / 2} .
$$

It should be noted that another construction could be performed in using the economy-size QR decomposition (see for instance [68]), which is also computationally efficient for $n \ll N$ with $N$ very large.

A parameterization $[W]=\mathcal{R}_{s, V}([A])$, defined on $\mathbb{M}_{N, n}$, of $\mathbb{S}_{N, n}$ at a given point $[V]$ in $\mathbb{S}_{N, n}$ is constructed in substituting the parameterization of $[Z]$ defined by Eq. (25) into $[W]=R_{s, V}([Z])$ that is defined by Eqs. (28) and (29). Consequently, the mapping $[A] \mapsto \mathcal{R}_{s, V}([A])$ from $\mathbb{M}_{N, n}$ into $\mathbb{S}_{N, n}$ is defined, for all $[A]$ in $\mathbb{M}_{N, n}$, by

$$
[W]=\mathcal{R}_{s, V}([A]):=R_{s, V}([A]-[V][D]) \quad, \quad[D]=\left([V]^{T}[M][A]+[A]^{T}[M][V]\right) / 2,
$$

and consequently, we have

$$
[W]^{T}[M][W]=\left[I_{n}\right] \quad, \quad[V]=\mathcal{R}_{s, V}\left(\left[0_{N, n}\right]\right) \in \mathbb{S}_{N, n} .
$$

\subsection{Construction of a parameterization of the subset $\mathcal{S}_{N, n}$ of the compact Stiefel manifold $\mathbb{S}_{N, n}$ induced by the additional constraint $[B]^{T}[W]=\left[0_{N_{\mathrm{cD}}, n}\right]$}

We have now to construct a parameterization $[W]=\mathcal{R}_{s, V}([A])$ in presence of an additional constraint equation $[B]^{T}[W]=\left[0_{N_{\mathrm{cD}}, n}\right]$ on $[W]$. Let us consider the case for which the ROB $[V]$ belongs to the subset $\mathcal{S}_{N, n}$ of $\mathbb{S}_{N, n}$ defined by

$$
\mathcal{S}_{N, n}=\left\{[V] \in \mathbb{M}_{N, n},[V]^{T}[M][V]=\left[I_{n}\right],[B]^{T}[V]=\left[0_{N_{\mathrm{cD}}, n}\right]\right\} \subset \mathbb{S}_{N, n},
$$

in which $0<N_{\text {с }}<N$ is the number of constraint equations and where $[B]$ is a given matrix such that

$$
[B] \in \mathbb{M}_{N, N_{\mathrm{CD}}} \quad, \quad[B]^{T}[B]=\left[I_{N_{\mathrm{cD}}}\right] .
$$

Such a parameterization is given by Eq. (30), in which $[A]$ is any matrix that belongs to the subset $\mathbb{M}_{N, n}^{B}$ of $\mathbb{M}_{N, n}$ defined by

$$
\mathbb{M}_{N, n}^{B}=\left\{[A] \in \mathbb{M}_{N, n},[A]=\left(\left[I_{N}\right]-[B][B]^{T}\right)[U],[U] \in \mathbb{M}_{N, n},\right\} .
$$

It can easily be seen that, for all $[A]$ in $\mathbb{M}_{N, n}^{B}$, we have $[B]^{T}[A]=\left[0_{N_{\mathrm{cD}}, n}\right]$ and consequently, $[B]^{T}[W]=\left[0_{N_{\mathrm{cD}}, n}\right]$.

- From a numerical point of view, the parameterization of matrix $[A]$ defined by Eq. (34) can be rewritten as $[A]=[U]-[B]\left\{[B]^{T}[U]\right\}$ in which $[B]^{T}[U] \in \mathbb{M}_{N_{\mathrm{cD}}, n}$. Consequently, the assemblage of the big $(N \times N)$ matrix $[B][B]^{T}$ is never done.

- If the constraint $[B]^{T}[W]=\left[0_{N_{\mathrm{cD}}, n}\right]$ does not exist, then Eq. (34) is replaced by $[A]=[U]$ with $[U] \in \mathbb{M}_{N, n}$. 


\subsection{Construction of a stochastic reduced-order basis (SROB) associated with the ROB}

The construction of the SROB, associated with ROB $[V]$, is performed for $\mathbb{S}_{N, n}$ with the additional constraint equation $[B]^{T}[W]=\left[0_{N_{\mathrm{c}}, n}\right]$, which means that matrix $[A]$ belongs to $\mathbb{M}_{N, n}^{B}$. If the constraint equation $[B]^{T}[W]=\left[0_{N_{\mathrm{cD}}, n}\right]$ is removed, then the stochastic modeling presented hereinafter holds (simply write that $[A]=[U]$ ). Consequently, the stochastic modeling of matrix $[U]$ will be the same for the two cases (with or without the constraint equation).

For a given ROB that is represented by a matrix $[V]$ given in $\mathcal{S}_{N, n} \subset \mathbb{S}_{N, n}$ (thus $[B]^{T}[V]=$ $\left.\left[0_{N_{\mathrm{cD}}, n}\right]\right)$, the associated SROB consists in introducing a random matrix $[\mathbf{W}]$, defined on a probability space $(\Theta, \mathcal{T}, \mathcal{P})$, with values in $\mathcal{S}_{N, n}$, such that

$$
[\mathbf{W}]^{T}[M][\mathbf{W}]=\left[I_{n}\right] \text { a.s. } \quad, \quad[B]^{T}[\mathbf{W}]=\left[0_{N_{\mathrm{cD}}, n}\right] \text { a.s. },
$$

and possibly, verifying additional constraints that will be defined after. We then have to construct the probability measure $P_{[\mathbf{W}]}$ of the random matrix $[\mathbf{W}]$, on $\mathbb{M}_{N, n}$, for which its support is the manifold $\mathcal{S}_{N, n}$,

$$
\operatorname{supp} P_{[\mathbf{W}]}=\mathcal{S}_{N, n} \subset \mathbb{S}_{N, n} \subset \mathbb{M}_{N, n}
$$

The construction of such a probability measure on the manifold $\mathcal{S}_{N, n}$ requires the introduction of an adapted parameterization of $\mathcal{S}_{N, n}$ in order to release the difficulties induced by the support of the measure. Below, we give a construction of the SROB in three steps, and the available information is gradually introduced in the construction.

\subsubsection{Step 1 of the construction of the SROB (introducing a parameterization)}

The SROB is constructed by using the parameterization of $[W]$ defined by Eqs. (30) and (34). Random matrix $[\mathbf{W}]$ can then be written as

$$
[\mathbf{W}]=\mathcal{R}_{s, V}([\mathbf{A}])=R_{s, V}([\mathbf{A}]-[V][\mathbf{D}]) \quad \text { with } \quad[\mathbf{D}]=\left([V]^{T}[M][\mathbf{A}]+[\mathbf{A}]^{T}[M][V]\right) / 2,
$$

in which $[\mathbf{A}]$ is a random matrix defined on $(\Theta, \mathcal{T}, \mathcal{P})$, with values in subset $\mathbb{M}_{N, n}^{B}$ of $\mathbb{M}_{N, n}$,

$$
[\mathbf{A}]=\left(\left[I_{N}\right]-[B][B]^{T}\right)[\mathbf{U}]=[\mathbf{U}]-[B]\left\{[B]^{T}[\mathbf{U}]\right\}
$$

where the random matrix $[\mathbf{U}]=\left[\mathbf{U}^{1} \ldots \mathbf{U}^{n}\right]$ is defined on $(\Theta, \mathcal{T}, \mathcal{P})$, with values in $\mathbb{M}_{N, n}$, in which the columns of $[\mathbf{U}]$ are $n$ random vectors $\mathbf{U}^{1}, \ldots, \mathbf{U}^{n}$ with values in $\mathbb{R}^{N}$. The deterministic mapping $R_{s, V}$ (from tangent vector space $T_{V} \mathbb{S}_{N, n}$ of $\mathbb{S}_{N, n}$ at point $[V]$ into $\mathbb{S}_{N, n}$ ) is defined by Eqs. (28) and (29). Note that $[\mathbf{D}]$ is a random matrix with values in $\mathbb{M}_{n}^{S}$.

\subsubsection{Step 2 of the construction of the SROB (defining the available information)}

Taking into account Eqs. (36) and (37), the stochastic model of random matrix $[\mathbf{W}]$ and its generator are completely defined by the stochastic model and the generator of random matrix $[\mathbf{U}]$.

(i) The construction of the SROB is performed in order that the statistical fluctuations of random matrix $[\mathbf{W}]$ are around deterministic matrix $[V]$. Taking into account that $[\mathbf{W}]=[V]$ for $[\mathbf{A}]=$ $\left[0_{N, n}\right]$, random matrix $[\mathbf{A}]$ must be a centered random variable, which is satisfied if $[\mathbf{U}]$ is also a centered random matrix (due to Eq. (37)). Consequently, we must have,

$$
E\{[\mathbf{U}]\}=\left[0_{N, n}\right] .
$$


(ii) In order to minimize the number of hyperparameters in the stochastic model of the centered random matrix $[\mathbf{U}]$, the $N n(N n+1) / 2$ components of the fourth-order symmetric covariance tensor $\left\{\mathbb{C}_{j k j^{\prime} k^{\prime}}\right\}_{j k j^{\prime} k^{\prime}}$ of $\mathbb{M}_{N, n}$-valued random matrix $[\mathbf{U}]$ are not kept because $N$ can be very large, and consequently, the proposed stochastic model would be unusable. We therefore choose the following reduced parameterization for tensor $\mathbb{C}$, which exhibits only $1+n(n+1) / 2$ hyperparameters, such that, for all $j$ and $j^{\prime}$ in $\{1, \ldots, N\}$, and for all $k$ and $k^{\prime}$ in $\{1, \ldots, n\}$,

$$
\mathbb{C}_{j k j^{\prime} k^{\prime}}=E\left\{\mathbf{U}_{j k} \mathbf{U}_{j^{\prime} k^{\prime}}\right\}=\left[C_{N}(\beta)\right]_{j j^{\prime}}\left[c_{n}\right]_{k k^{\prime}}
$$

in which

- $\left[C_{N}(\beta)\right] \in \mathbb{M}_{N}^{+}$is a type-covariance matrix depending on a hyperparameter $\beta$ such that $0<\beta_{d} \leq \beta \leq \beta_{u}<+\infty$, for which its construction is detailed in [65]. This covariance matrix allows for introducing a correlation between the components $U_{1}^{k}, \ldots, U_{N}^{k}$ of each random vector $\mathbf{U}^{k}$ such that $U_{j}^{k}=[\mathbf{U}]_{j k}$.

- $\left[c_{n}\right] \in \mathbb{M}_{n}^{+}$is a type-covariance matrix and consequently, there is an upper triangular matrix $[\sigma]$ belonging to $\mathbb{M}_{n}^{u}$ such that (Cholesky's factorization),

$$
\left[c_{n}\right]=[\sigma]^{T}[\sigma] .
$$

Matrix $\left[c_{n}\right]$ allows for describing the correlation between the random vectors $\mathbf{U}^{1}, \ldots, \mathbf{U}^{n}$.

Using Eqs. (38), (39), and (40), the second-order $\mathbb{M}_{N, n}$-valued random matrix $[\mathbf{U}]$ can be rewritten as $[\mathbf{U}]=[\mathbf{G}][\sigma]$ in which $[\mathbf{G}]$ is a second-order centered $\mathbb{M}_{N, n}$-valued random matrix defined on probability space $(\Theta, \mathcal{T}, \mathcal{P})$, such that, for all $j$ and $j^{\prime}$ in $\{1, \ldots, N\}$, and for all $k$ and $k^{\prime}$ in $\{1, \ldots, n\}$,

$$
E\left\{\mathbf{G}_{j k}\right\}=0 \quad, \quad E\left\{\mathbf{G}_{j k} \mathbf{G}_{j^{\prime} k^{\prime}}\right\}=\left[C_{N}(\beta)\right]_{j j^{\prime}} \delta_{k k^{\prime}} .
$$

It can be seen that

$$
E\left\{[\mathbf{G}][\mathbf{G}]^{T}\right\}=n\left[C_{N}(\beta)\right] \quad, \quad E\left\{[\mathbf{G}]^{T}[\mathbf{G}]\right\}=\left(\operatorname{tr}\left[C_{N}(\beta)\right]\right)\left[I_{n}\right] .
$$

Consequently, for the construction proposed, random matrix $[\mathbf{U}]$ is parameterized as

$$
[\mathbf{U}]=[\mathbf{G}][\sigma],
$$

and is such that

$$
E\left\{[\mathbf{U}][\mathbf{U}]^{T}\right\}=\left(\operatorname{tr}\left[c_{n}\right]\right)\left[C_{N}(\beta)\right] \quad, \quad E\left\{[\mathbf{U}]^{T}[\mathbf{U}]\right\}=\left(\operatorname{tr}\left[C_{N}(\beta)\right]\right)\left[c_{n}\right]
$$

\subsubsection{Step 3 of the construction of the SROB (constructing the stochastic model of $[W]$ )}

(i) About the stochastic model of random matrix $[\boldsymbol{G}]$. The stochastic model of the second-order centered random matrix $[\mathbf{G}]$ with values in $\mathbb{M}_{N, n}$ and its generator of independent realizations is detailed in [65] for which $[\mathbf{G}]$ is a non-Gaussian random matrix. This construction is based on the finite element discretization of a non-Gaussian random field whose trajectories are indefinitely continuously differentiable functions almost surely in order to preserve the regularity properties of the solution of the HFM. The stochastic model proposed is chosen in order that it exhibits only a scalar hyperparameter $\beta$ and does not require the explicit construction of the big matrix $\left[C_{N}(\beta)\right]$ for which the number of entries is $N(N+1) / 2$, that is unusable for a large 
value of $N$.

(ii) Stochastic model and hyperparameters of random matrix $[\boldsymbol{W}]$. The stochastic representation of the second-order non-Gaussian and not centered random matrix $[\mathbf{W}]$ with values in the manifold $\mathcal{S}_{N, n} \subset \mathbb{S}_{N, n} \subset \mathbb{M}_{N, n}$ is defined by Eqs. (36), (37), and (42), which are rewritten (using Eqs. (28) and (29)) as

$$
\begin{aligned}
{[\mathbf{W}] } & =R_{s, V}([\mathbf{Z}])=([V]+s[\mathbf{Z}])\left[H_{s}(\mathbf{Z})\right], \\
{\left[H_{s}(\mathbf{Z})\right] } & =\left(\left[I_{n}\right]+s^{2}[\mathbf{Z}]^{T}[M][\mathbf{Z}]\right)^{-1 / 2}, \\
{[\mathbf{Z}] } & =[\mathbf{A}]-[V][\mathbf{D}] \\
{[\mathbf{D}] } & =\left([V]^{T}[M][\mathbf{A}]+[\mathbf{A}]^{T}[M][V]\right) / 2, \\
{[\mathbf{A}] } & =[\mathbf{U}]-[B]\left\{[B]^{T}[\mathbf{U}]\right\}, \\
{[\mathbf{U}] } & =[\mathbf{G}(\beta)][\sigma] .
\end{aligned}
$$

in which

- $[\mathbf{G}(\beta)]$ is the second-order non-Gaussian centered random matrix with values in $\mathbb{M}_{N, n}$ detailed in [65], and for which the covariance tensor is defined by Eq. (41).

- $[\sigma]$ is a given upper triangular matrix in $\mathbb{M}_{n}^{u}$ (positive diagonal entries).

- $[B]$ is a given matrix in $\mathbb{M}_{N, N_{\mathrm{CD}}}$ such that $[B]^{T}[B]=\left[I_{N_{\mathrm{CD}}}\right]$.

- $[V]$ is a given matrix in $\mathcal{S}_{N, n}$.

For $[V]$ fixed in $\mathcal{S}_{N, n}$, the $2+n(n+1) / 2$ hyperparameters of the stochastic model of random matrix $[\mathbf{W}]$ with values in $\mathcal{S}_{N, n}$ are:

- the deterministic real parameter $s$ is such that $\varepsilon_{0} \leq s \leq 1$ in which $\varepsilon_{0}$ is given and such that $0 \leq \varepsilon_{0}<1$ (if $s=0$, then $[\mathbf{W}]=[V]$ is deterministic and there are no statistical fluctuations),

- the deterministic real parameter $\beta$ such that $0<\beta_{d} \leq \beta \leq \beta_{u}<+\infty$ in which $\beta_{d}$ and $\beta_{u}$ are given,

- the upper triangular matrix $[\sigma]$ in $\mathbb{M}_{n}^{\mathrm{u}}$ (positive diagonal entries), which is parameterized by $n(n+1) / 2$ parameters, and such that the diagonal entries satisfy the constraints $\varepsilon_{0} \leq$ $[\sigma]_{11}, \ldots,[\sigma]_{n n} \leq \sigma_{u}<+\infty$, in which $\sigma_{u}$ is given.

The hyperparameter is thus $\boldsymbol{\alpha}=\left(s, \beta,\left\{[\sigma]_{k k^{\prime}}, 1 \leq k \leq k^{\prime} \leq n\right\}\right)$ with length $m_{\boldsymbol{\alpha}}=2+n(n+$ $1) / 2$, which belongs to the admissible set $\mathcal{C}_{\alpha}$ defined by

$$
\mathcal{C}_{\boldsymbol{\alpha}}=\left\{s \in\left[\varepsilon_{0}, 1\right], \beta \in\left[\beta_{d}, \beta_{u}\right], \varepsilon_{0} \leq[\sigma]_{11}, \ldots,[\sigma]_{n n} \leq \sigma_{u},[\sigma]_{k k^{\prime}} \in \mathbb{R}, k<k^{\prime}\right\} .
$$

\section{APPLICATION IN NONLINEAR COMPUTATIONAL STRUCTURAL DYNAMICS}

In this section, a verificaton problem for the theory exposed above is presented. The chosen HFM corresponds to a finite element model of a three-dimensional slender damped elastic bounded medium with nonlinear barriers that induce nonlinearities in the dynamical system. Additional applications for a more deeper validation of the proposed theory can be found in 
[65]. The problem is intentionally chosen to be sufficiently simple so that it can be easily reproduced by the interested reader. Nevertheless, it is representative of difficult problems as far as ROM model form uncertainties are concerned. In the frequency domain, the excitation has an energy located in a narrow frequency band within in a broad frequency band of analysis. The differences between the HFM and the ROM are very small in the frequency band of excitation (small uncertainties), but are large outside this frequency band (large uncertainties). These large uncertainties are due to the transfer of the energy outside the frequency band of excitation due to the nonlinearities in the dynamical system. Consequently, they correspond to second-order contributions. This application shows how such second-order contributions, which are located outside the frequency band of excitation, can be predicted using the nonparametric probabilistic approach of model uncertainties.

\subsection{Description of the mechanical system}

The mechanical system is made up of a 3D linear elastic structure with two elastic barriers that induce impact non-linearities. It is defined in a cartesian coordinate system $O x_{1} x_{2} x_{3}$ (see Fig. (1). The cylinder has length $L_{1}=1.20 \mathrm{~m}$ and a rectangular section with height $L_{2}=0.12 \mathrm{~m}$ and width $L_{3}=0.24 \mathrm{~m}$. The two end sections are located at $x_{1}=0$ and $x_{1}=L_{1}$. The elastic medium is made of a homogeneous and isotropic elastic material for which the Young modulus is $10^{10} \mathrm{~N} / \mathrm{m}^{2}$, the Poisson coefficient is 0.15 and the mass density is $1500 \mathrm{Kg} / \mathrm{m}^{3}$. A damping term is added and is described by a global damping rate of $\xi_{d}=0.01$ for each elastic mode of the structure without the elastic barriers, and will be introduced at the ROM level. The nonlinear

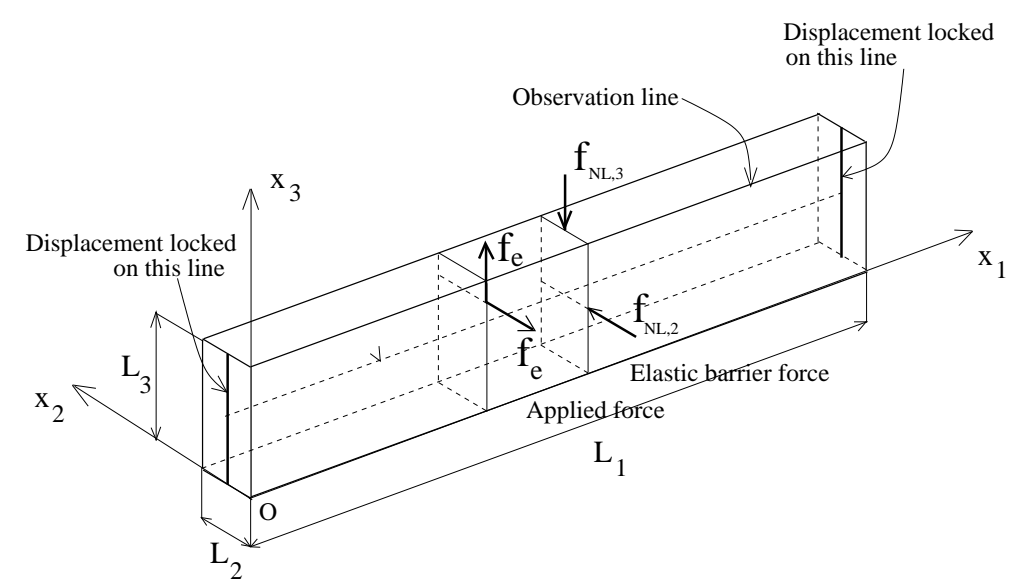

Figure 1: Scheme of the mechanical system: slender elastic structure with elastic barriers.

forces are due to elastic barriers that induce two nonlinear point forces on the structure, one in direction $x_{2}$ applied to the point $\left(x_{1}=0.66, x_{2}=0, x_{3}=L_{3} / 2\right)$, and another one in direction $x_{3}$ applied to the point $\left(x_{1}=0.66, x_{2}=L_{2} / 2, x_{3}=L_{3}\right)$ (see Fig. 1) of intensity $-f_{\mathrm{NL}, 2}$ and $-f_{\mathrm{NL}, 3}$ that are assumed to be independent of the velocity and such that

$$
\begin{gathered}
f_{\mathrm{NL}, 2}(\eta)=k_{b, 2}\left(\eta+\varepsilon_{b, 2}\right) \mathbb{1}_{\mathbb{R}^{+}}\left(-\eta-\varepsilon_{b, 2}\right) \quad, \quad \eta \in \mathbb{R}, \\
f_{\mathrm{NL}, 3}(\eta)=k_{b, 3}\left(\zeta-\varepsilon_{b, 3}\right) \mathbb{1}_{\mathbb{R}^{+}}\left(\zeta-\varepsilon_{b, 3}\right) \quad, \quad \zeta \in \mathbb{R},
\end{gathered}
$$

where $k_{b, 2}=k_{b, 3}=2 \times 10^{9} \mathrm{~N} / \mathrm{m}$ is the elasticity constant of the barriers and $\varepsilon_{b, 2}=\varepsilon_{b, 3}=$ $2 \times 10^{-4} \mathrm{~m}$ are the two gaps (positive values). A time-dependent point force is applied at the 
boundary of the slender structure at the point $\left(x_{1}=0.46, x_{2}=0, x_{3}=0.2\right)$ (see Fig. 1) in the directions $x_{2}$ and $x_{3}$. The $x_{2}$ - and $x_{3}$-components of this point force are equal to a square integrable real-valued function $f_{e}$ defined by

$$
f_{e}(t)=\frac{f_{0}}{\pi t}\left\{\sin \left(t\left(\omega_{c}+\Delta \omega_{c} / 2\right)\right)-\sin \left(t\left(\omega_{c}-\Delta \omega_{c} / 2\right)\right\} \quad, \quad t \in\left[t_{0}, T\right],\right.
$$

where $f_{0}=100 \mathrm{~N}$ is the amplitude, $\omega_{c}=2 \pi \times 470 \mathrm{rad} / \mathrm{s}$ is the central frequency band, and $\Delta \omega_{c}=2 \pi \times 300 \mathrm{rad} / \mathrm{s}$ is the bandwidth. The signal energy of this excitation is mainly concentrated in the frequency band $[320,620] \mathrm{Hz}$. An observation line of equation $\left\{0 \leq x_{1} \leq\right.$ $\left.L_{1} ; x_{2}=0 ; x_{3}=L_{3}\right\}$ for $t \in\left[t_{0}, T\right]$ is introduced. In order to limit the number of figures, $\mathrm{o}$ nly the $x_{2}$ - and $x_{3}$-displacements of 2 observation points belonging to the observation line (see Fig. (1) are considered and are denoted by $\mathrm{Obs}_{34}$ and $\mathrm{Obs}_{51}$ for which the $x_{1}$-coordinates are 0.66 and 1.00. At initial time $t_{0}=-0.0403 \mathrm{~s}$, the system is at rest. The final time is $T=0.3790 \mathrm{~s}$. The frequency band of observation is chosen as $\mathcal{B}_{o}=\left[0, \omega_{o}\right]$ with $\omega_{o}=2 \pi \times 1550 \mathrm{rad} / \mathrm{s}$. The time sampling is defined by the frequency $\omega_{\max }=2 \pi \times 12,400 \mathrm{rad} / \mathrm{s}$. There are 10,400 time steps and the sampling time step is $\Delta t=\pi / \omega_{\max }=4.032 \times 10^{-5} \mathrm{~s}$. There are 10,400 frequency steps in the frequency band $\left[-\omega_{\max }, \omega_{\max }\right]$ and the sampling frequency step is $\Delta \omega=$ $2 \pi \times 2.38 \mathrm{rad} / \mathrm{s}$.

\subsection{HFM, numerical solver, and results}

(i) High fidelity computational model. A 3D computational model is constructed with a finite element mesh made up of $60 \times 6 \times 12=4320$ three-dimensional 8-nodes solid elements. There are 5551 nodes and $N=16,653$ dofs. The number of zero Dirichlet conditions is $N_{\text {cD }}=78$ (the displacements are zero for $2 \times 13$ nodes). For this HFM, the equations Eqs. (1) to (4) are rewritten as follows:

$$
\begin{aligned}
& \left.[M] \ddot{\mathbf{y}}(t)+\mathbf{g}(\mathbf{y}(t), \dot{\mathbf{y}}(t))=\mathbf{f}(t) \quad, \quad t \in] t_{0}, T\right], \\
& \mathbf{g}(\mathbf{y}(t), \dot{\mathbf{y}}(t))=[\mathbb{D}] \dot{\mathbf{y}}(t)+[K] \mathbf{y}(t)+\mathbf{f}_{\mathrm{NL}}(\mathbf{y}(t)),
\end{aligned}
$$

with the zero initial conditions at time $t_{0}$,

$$
\mathbf{y}\left(t_{0}\right)=\mathbf{0}_{N} \quad, \quad \dot{\mathbf{y}}\left(t_{0}\right)=\mathbf{0}_{N},
$$

and the $N_{\text {с }}<N$ constraint equations written in matrix form as

$$
[B]^{T} \mathbf{y}(t)=\mathbf{0}_{N_{\mathrm{CD}}} \quad, \quad t \in\left[t_{0}, T\right],
$$

where $[B]$ is a given matrix in $\mathbb{M}_{N, N_{\mathrm{CD}}}$ such that $[B]^{T}[B]=\left[I_{N_{\mathrm{cD}}}\right]$, which is constructed by using the $N_{\text {с }}$ zero Dirichlet conditions defined in Section 4.1. A construction of $[\mathbb{D}]$ is detailed in [65] for which the numerical complexity of the product of $[\mathbb{D}]$ by a vector in $\mathbb{R}^{N}$ is of the order of the one corresponding to the product of $[M]$ by a vector in $\mathbb{R}^{N}$, which is much less than $N^{2}$ because $[M]$ is a sparse finite element matrix. In the frequency band $\mathcal{B}_{o}=\left[0, \omega_{o}\right]$, the QoI is the vector $\widehat{\mathbf{o}}(\omega)=\left(\widehat{o}_{1}(\omega), \ldots, \widehat{o}_{m_{\mathbf{0}}}(\omega)\right) \in \mathbb{C}^{m_{\mathbf{0}}}$ defined as follows:

- For plotting and analyzing the responses of the SROM, $m_{\mathbf{0}}=2 \times 2=4$ dofs in direction $x_{2}$ and $x_{3}$ of the 2 observation nodes $\mathrm{Obs}_{34}$ and $\mathrm{Obs}_{51}$. 
- For the identification of the hyperparameter $\boldsymbol{\alpha}$ of the SROB, $m_{\mathbf{0}}=2 \times 61=122$ dofs in direction $x_{2}$ and $x_{3}$ of all the 61 nodes belonging to the observation line.

For all $\omega$ in $\mathcal{B}_{o}$, the complex vector vector $\widehat{\mathbf{o}}(\omega)$ is written as

$$
\widehat{\mathbf{o}}(\omega)=\mathbf{h}\left(-\omega^{2} \widehat{\mathbf{y}}(\omega)\right) \quad, \quad \omega \in \mathcal{B}_{o},
$$

in which $\mathbf{h}$ is a linear mapping from $\mathbb{C}^{N}$ into $\mathbb{C}^{m_{\mathbf{0}}}$, which extracts the dofs from vector $-\omega^{2} \widehat{\mathbf{y}}(\omega) \in$ $\mathbb{C}^{N}$, and where

$$
\widehat{\mathbf{y}}(\omega)=\int_{t_{0}}^{T} e^{-i \omega t} \mathbf{y}(t) d t \quad, \quad \omega \in \mathcal{B}_{o} .
$$

(ii) Numerical solver. The implicit Newmark time-integration scheme [69] is used (with parameters $\delta=0.5$ and $\alpha=0.25(0.5+\delta)^{2}=0.25$ ) and with a fixed point method (without relaxation) at each sampling time with a relative precision of $10^{-6}$. In order to guaranty the convergence of the fixed point method, a local adaptive time step is implemented (time step $\Delta t$ is locally decreased). Such a numerical method has been preferred to the other possible ones due to the presence of contacts induced by the barriers, which require an adaptive time steps.

(iii) Results and quantification of the effects of the nonlinearities. In Fig. 2, the four figures compare the graphs $\nu \mapsto \log _{10}\left(\left|\widehat{o}_{j}(2 \pi \nu)\right|\right)$ computed with the HFM, with the graphs computed with the linear HFM (that is to say, in removing the nonlinear elastic barriers in the HFM), for the $x_{2}$ - and $x_{3}$-accelerations of $\mathrm{Obs}_{51}$ and $\mathrm{Obs}_{34}$. These figures show the effects of the nonlinear elastic barriers on the responses, in particular, it can be seen an important transfer of the energy in the frequency band that is outside the main frequency band $[320,620] \mathrm{Hz}$ of the excitation.

\subsection{ROM, numerical solver, and results}

(i) Reduced-order computational model. Let $\left\{\varphi^{1}, \ldots, \varphi^{n}\right\}$ be the first $n$ elastic modes associated with the first $n$ eigenfrequencies $0<\omega_{1}<\ldots<\omega_{n}$ of the linear undamped structure associated with the nonlinear damped dynamical system, which are such that

$$
[K] \varphi^{k}=\lambda_{k}[M] \varphi^{k}
$$

with the constraint equation

$$
[B]^{T} \boldsymbol{\varphi}^{k}=\mathbf{0}_{N_{\mathrm{CD}}} \quad, \quad k=1, \ldots, n,
$$

and where $\lambda_{1}=\omega_{1}^{2}, \ldots, \lambda_{n}=\omega_{n}^{2}$. The elastic modes satisfy the usual orthogonality properties,

$$
<[M] \varphi^{k}, \varphi^{k^{\prime}}>=\delta_{k k^{\prime}} \quad, \quad<[K] \varphi^{k}, \varphi^{k^{\prime}}>=\lambda_{k} \delta_{k k^{\prime}} .
$$

For the nonlinear dynamical system, the ROB is chosen as $[V]=\left[\varphi^{1} \ldots \varphi^{n}\right] \in \mathbb{M}_{N, n}$, which is such that

$$
[V]^{T}[M][V]=\left[I_{n}\right] \quad, \quad[B]^{T}[V]=\left[0_{N_{\mathrm{cD}}, n}\right] .
$$

Using the ROB $[V] \in \mathbb{M}_{N, n}$ and Eq. (63), the ROM associated with the HFM defined by Eqs. (54) to (57) is written as

$$
\begin{gathered}
\mathbf{y}^{(n)}(t)=[V] \mathbf{q}(t) \quad, \quad t \in\left[t_{0}, T\right] \\
\left.\left.\ddot{\mathbf{q}}(t)+[V]^{T}[\mathbb{D}][V] \dot{\mathbf{q}}(t)+[V]^{T}[K][V] \mathbf{q}(t)+[V]^{T} \mathbf{f}_{\mathrm{NL}}([V] \mathbf{q}(t))=[V]^{T} \mathbf{f}(t), t \in\right] t_{0}, T\right],
\end{gathered}
$$



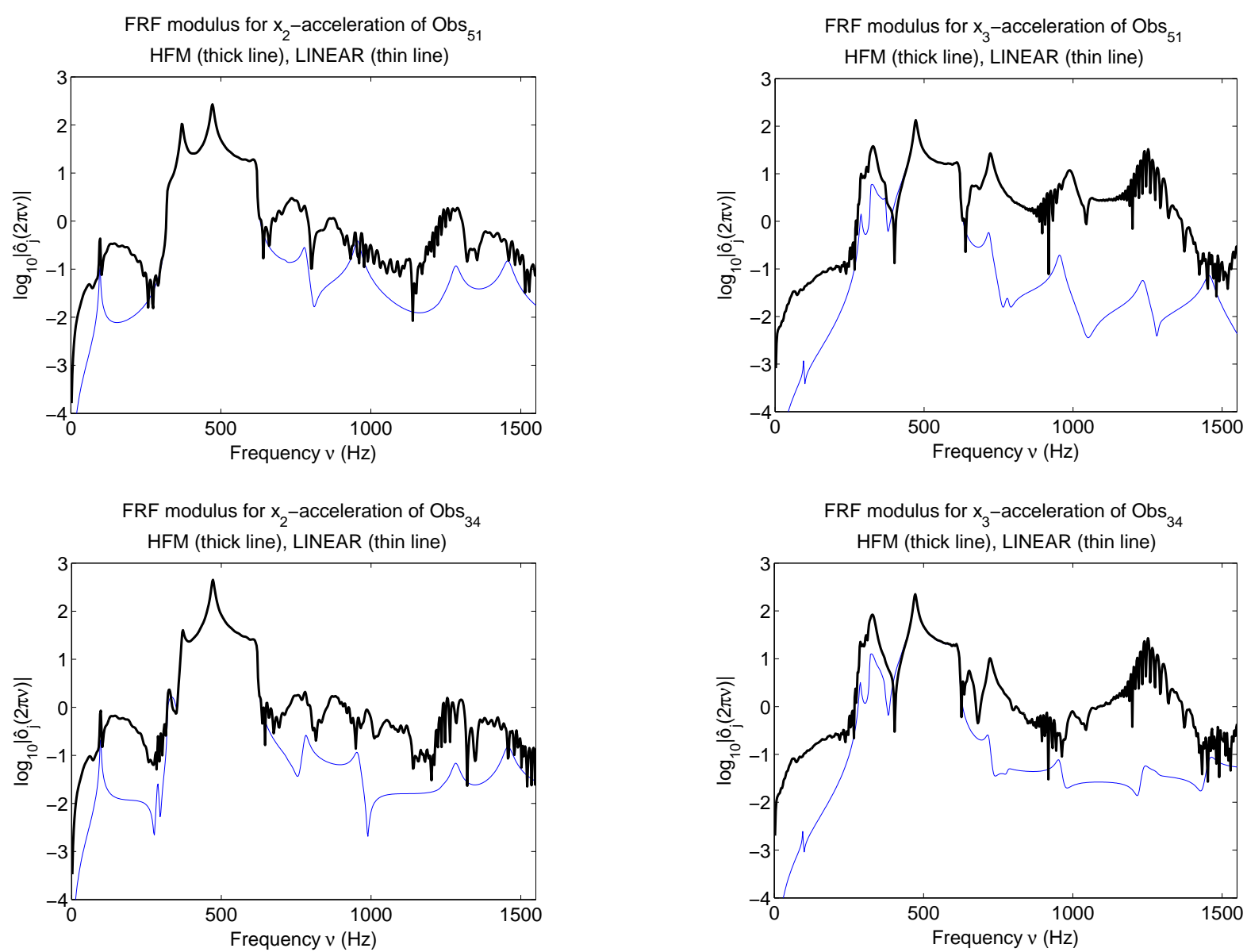

Figure 2: Graphs $\nu \mapsto \log _{10}\left(\left|\widehat{o}_{j}(2 \pi \nu)\right|\right)$ computed with the HFM (thick lines) and with the LINEAR HFM (thin lines) for $x_{2}$ - and $x_{3}$-accelerations of $\mathrm{Obs}_{51}$ (up left and up right figures), and for $x_{2}$ - and $x_{3}$-accelerations of $\mathrm{Obs}_{34}$ (down left and down right figures).

with the initial conditions

$$
\mathbf{q}\left(t_{0}\right)=\mathbf{0}_{n} \quad, \quad \dot{\mathbf{q}}\left(t_{0}\right)=\mathbf{0}_{n} .
$$

The approximation $\widehat{\mathbf{o}}^{(n)}(\omega)$ of the QoI $\widehat{\mathbf{o}}(\omega)$ defined by Eqs. (58) and (59) is written as

$$
\begin{gathered}
\widehat{\mathbf{o}}^{(n)}(\omega)=\mathbf{h}\left(-\omega^{2} \widehat{\mathbf{y}}^{(n)}(\omega)\right) \quad, \quad \omega \in \mathcal{B}_{o}, \\
\widehat{\mathbf{y}}^{(n)}(\omega)=\int_{t_{0}}^{T} e^{-i \omega t} \mathbf{y}^{(n)}(t) d t \quad, \quad \omega \in \mathcal{B}_{o} .
\end{gathered}
$$

(ii) Numerical solver. The algorithm described in Section 4.2-(ii) is used but is adapted to Eq. (65) that is rewritten as $\ddot{\mathbf{q}}(t)+2 \xi_{d}\left[\lambda^{(n)}\right]^{1 / 2} \dot{\mathbf{q}}(t)+\left[\lambda^{(n)}\right] \mathbf{q}(t)=[V]^{T} \mathbf{f}(t)-[V]^{T} \mathbf{f}_{\mathrm{NL}}([V] \mathbf{q}(t))$ in which $\left[\lambda^{(n)}\right]$ is the positive-definite diagonal matrix whose diagonal entries are $\lambda_{1}, \ldots, \lambda_{n}$.

(iii) Choice of the reduced-order dimension n, results, and quantification of the errors induced by the use of the ROM instead of the HFM. Taking into account that a significative difference between the responses computed with ROM and with the HFM must be generated (in order to validate the capability of the nonparametric stochastic method proposed to take into account this type of modeling errors), a good compromise between the numerical cost and 
the quality of the ROM leads us to choose $n=20$ as the dimension of the ROM. We then have $\omega_{1}=2 \pi \times 96.69 \mathrm{rad} / \mathrm{s}, \omega_{4}=2 \pi \times 472 \mathrm{rad} / \mathrm{s}, \omega_{5}=2 \pi \times 720 \mathrm{rad} / \mathrm{s}, \omega_{11}=2 \pi \times 1474 \mathrm{rad} / \mathrm{s}$, $\omega_{12}=2 \pi \times 1754 \mathrm{rad} / \mathrm{s}$, and $\omega_{20}=2 \pi \times 2936 \mathrm{rad} / \mathrm{s}$. Consequently, there are 4 elastic modes in the frequency band $[0,620] \mathrm{Hz}$ containing the main part of the excitation, 11 elastic modes in the frequency band of observation $[0,1550] \mathrm{Hz}$ and 9 elastic modes in the frequency band $[1550,3100] \mathrm{Hz}$. In Fig. 3, the four figures show a comparison of the graphs $\nu \mapsto \log _{10}\left(\left|\widehat{o}_{j}(2 \pi \nu)\right|\right)$ computed with the HFM, with the graphs $\nu \mapsto \log _{10}\left(\left|\widehat{o}_{j}^{(n)}(2 \pi \nu)\right|\right)$ computed with the ROM, for the $x_{2}$ - and $x_{3}$-accelerations of $\mathrm{Obs}_{51}$ and $\mathrm{Obs}_{34}$. These figures show that the differences between the HFM and the ROM are very small in the frequency band $[320,620] \mathrm{Hz}$ of the excitation, but are significant outside this frequency band. Such differences could be reduced in increasing dimension $n$ of the ROM, but as we have explained above, the reduced-order dimension $n$ is chosen in order that significant differences exist between the ROM and the HFM.
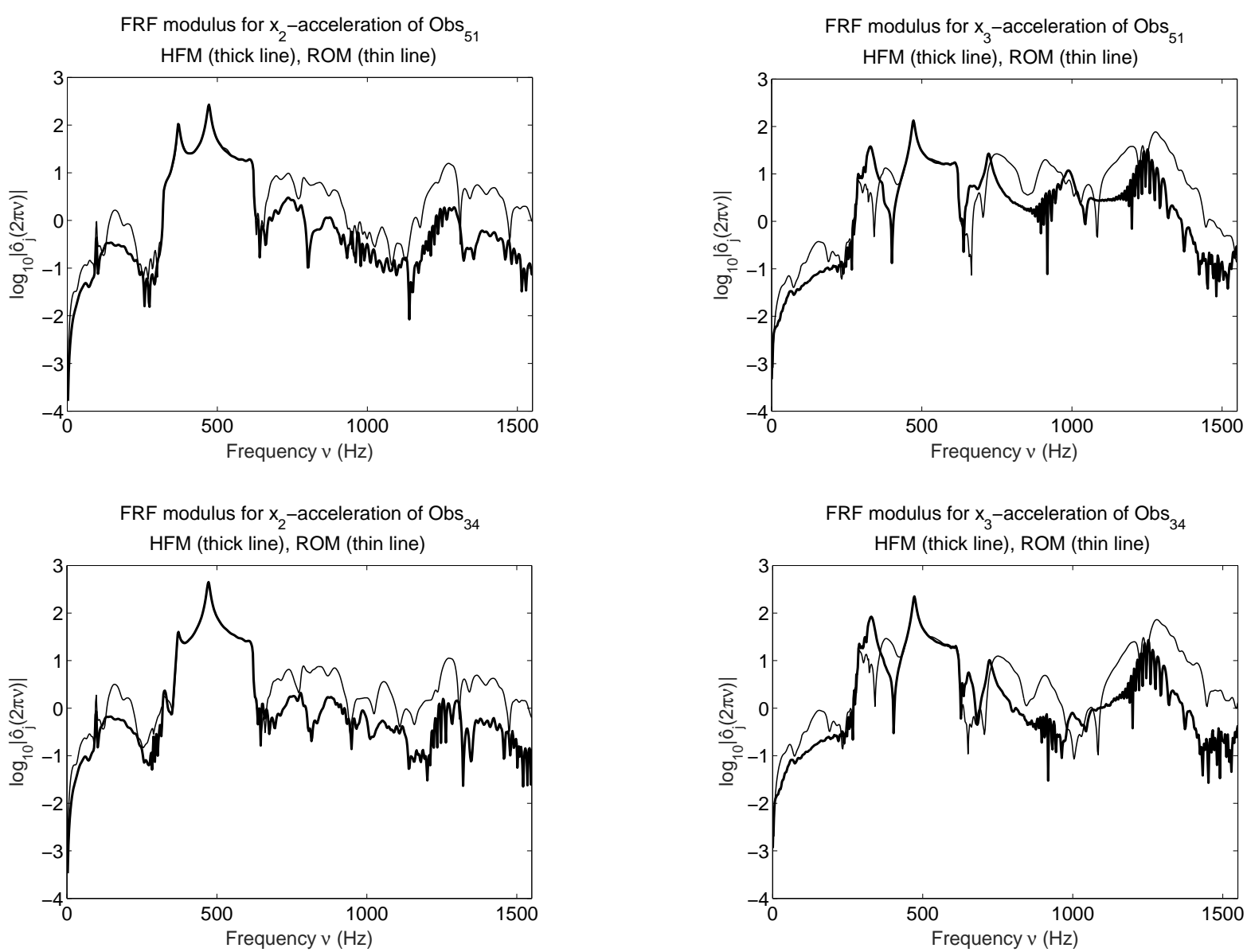

Figure 3: Graphs $\nu \mapsto \log _{10}\left(\left|\widehat{o}_{j}(2 \pi \nu)\right|\right)$ computed with the HFM (thick lines) and graphs $\nu \mapsto \log _{10}\left(\left|\widehat{o}_{j}^{(n)}(2 \pi \nu)\right|\right)$ computed with the ROM (thin lines) for $x_{2}$ - and $x_{3}$-accelerations of $\mathrm{Obs}_{51}$ (up left and up right figures), and for $x_{2}$ - and $x_{3}$-accelerations of $\mathrm{Obs}_{34}$ (down left and down right figures).

\subsection{SROM, stochastic solver, and results}

(i) Stochastic reduced-order computational model. From Eqs. (11) to (14), the SROM associated with Eqs. (64) to (68), is obtained in replacing $[V]$ by the random matrix $[\mathbf{W}]$. Conse- 
quently, $\mathbf{y}^{(n)}(t), \mathbf{q}(t)$, and $\widehat{\mathbf{o}}^{(n)}(\omega)$, become the random vectors $\mathbf{Y}^{(n)}(t), \mathbf{Q}(t)$, and $\widehat{\mathbf{O}}^{n}(\omega)$, and the SROM is written as

$$
\begin{gathered}
\mathbf{Y}^{(n)}(t)=[\mathbf{W}] \mathbf{Q}(t) \quad, \quad t \in\left[t_{0}, T\right] \\
\left.\left.\ddot{\mathbf{Q}}(t)+[\mathbf{W}]^{T}[\mathbb{D}][\mathbf{W}] \dot{\mathbf{Q}}(t)+[\mathbf{W}]^{T}[K][\mathbf{W}] \mathbf{Q}(t)+[\mathbf{W}]^{T} \mathbf{f}_{\mathrm{NL}}([\mathbf{W}] \mathbf{Q}(t))=[\mathbf{W}]^{T} \mathbf{f}(t), t \in\right] t_{0}, T\right],
\end{gathered}
$$

with the initial conditions

$$
\mathbf{Q}\left(t_{0}\right)=\mathbf{0}_{n} \quad, \quad \dot{\mathbf{Q}}\left(t_{0}\right)=\mathbf{0}_{n} \quad \text {, a.s } .
$$

Let $\widehat{\mathbf{O}}^{(n)}(\omega ; \boldsymbol{\alpha})$ be the random QoI with values in $\mathbb{C}^{m_{o}}$ with $m_{o}=122$, depending on hyperparameter $\boldsymbol{\alpha}$, such that

$$
\begin{gathered}
\widehat{\mathbf{O}}^{(n)}(\omega ; \boldsymbol{\alpha})=\mathbf{h}\left(-\omega^{2} \widehat{\mathbf{Y}}^{(n)}(\omega ; \boldsymbol{\alpha})\right) \quad, \quad \omega \in \mathcal{B}_{o}, \\
\widehat{\mathbf{Y}}^{(n)}(\omega ; \boldsymbol{\alpha})=\int_{t_{0}}^{T} e^{-i \omega t} \mathbf{Y}^{(n)}(t ; \boldsymbol{\alpha}) d t \quad, \quad \omega \in \mathcal{B}_{o} .
\end{gathered}
$$

(ii) Identification of the hyperparameter. The problem is the identification of hyperparameter $\boldsymbol{\alpha}=(s, \beta, \sigma)$ that is defined in Section 3.3.3-(ii), with length $m_{\boldsymbol{\alpha}}=2+n(n+1) / 2=212$, and which belongs to the admissible set $\mathcal{C}_{\alpha}=\mathbb{R}^{+} \times[0.01,0.1] \times \mathbb{M}_{n}^{\mathrm{u}}$. For defining the cost function $J(\boldsymbol{\alpha})$, we introduce the random function $\omega \mapsto \mathbf{d B}^{(n)}(\omega ; \boldsymbol{\alpha})=\left(\mathrm{dB}_{1}^{(n)}(\omega ; \boldsymbol{\alpha}), \ldots, \mathrm{dB}_{m_{o}}^{(n)}(\omega ; \boldsymbol{\alpha})\right)$ defined on $\mathcal{B}_{o}$ with valued in $\mathbb{R}^{m_{o}}$ such that, for all $j=1, \ldots, m_{o}$ (with $\left.m_{o}=122\right)$,

$$
\mathrm{dB}_{j}^{(n)}(\omega ; \boldsymbol{\alpha})=\log _{10}\left(\left|\widehat{O}_{j}^{(n)}(\omega ; \boldsymbol{\alpha})\right|\right),
$$

in which $\widehat{\mathbf{O}}^{(n)}(\omega ; \boldsymbol{\alpha})=\left(\widehat{O}_{1}^{(n)}(\omega ; \boldsymbol{\alpha}), \ldots, \widehat{O}_{m_{o}}^{(n)}(\omega ; \boldsymbol{\alpha})\right)$ is defined by Eq. (72). In order to define the target functions for constructing the cost function, we introduce the functions $\omega \mapsto$ $\mathbf{d b}^{\text {ref }}(\omega)=\left(\mathrm{db}_{1}^{\text {ref }}(\omega), \ldots, \mathrm{db}_{m_{o}}^{\text {ref }}(\omega)\right)$ and $\omega \mapsto \mathbf{d b}^{(n)}(\omega)=\left(\mathrm{db}_{1}^{(n)}(\omega), \ldots, \mathrm{db}_{m_{o}}^{(n)}(\omega)\right)$, defined on $\mathcal{B}_{o}$ with values in $\mathbb{R}^{m_{o}}$ such that, for all $j=1, \ldots, m_{o}$,

$$
\mathrm{db}_{j}^{\text {ref }}(\omega)=\log _{10}\left(\left|\widehat{o}_{j}(\omega)\right|\right) \quad, \quad \mathrm{db}_{j}^{(n)}(\omega)=\log _{10}\left(\left|\widehat{o}_{j}^{(n)}(\omega)\right|\right),
$$

in which $\widehat{\mathbf{o}}(\omega)=\left(\widehat{o}_{1}(\omega), \ldots, \widehat{o}_{m_{o}}(\omega)\right)$ is defined by Eq. $\left(\underline{58)}\right.$ and where $\widehat{\mathbf{o}}^{(n)}(\omega)=\left(\widehat{o}_{1}^{(n)}(\omega), \ldots\right.$, $\left.\widehat{O}_{m_{o}}^{(n)}(\omega)\right)$ is defined by Eq. (67). The cost function is defined by Eq. (15) with

$$
J(\boldsymbol{\alpha})=w_{J} J_{\text {mean }}(\boldsymbol{\alpha})+\left(1-w_{J}\right) J_{\text {std }}(\boldsymbol{\alpha}),
$$

in which

$$
\begin{gathered}
J_{\text {mean }}(\boldsymbol{\alpha})=\frac{1}{c_{\text {mean }}} \sum_{j=1}^{m_{o}} \int_{\mathcal{B}_{o}}\left|\mathrm{db}_{j}^{\text {ref }}(\omega)-E\left\{\mathrm{~dB}_{j}^{(n)}(\omega ; \boldsymbol{\alpha})\right\}\right|^{2} w_{j}(\omega) d \omega, \\
J_{\text {std }}(\boldsymbol{\alpha})=\frac{1}{c_{\text {std }}} \sum_{j=1}^{m_{o}} \int_{\mathcal{B}_{o}}\left|v_{j}^{(\text {ref }, n)}(\omega)-v_{j}^{(n)}(\omega ; \boldsymbol{\alpha})\right|^{2} w_{j}(\omega) d \omega
\end{gathered}
$$

in which the positive constants $c_{\text {mean }}$ and $c_{\text {std }}$ are defined by

$$
\left.c_{\text {mean }}=\sum_{j=1}^{m_{o}} \int_{\mathcal{B}_{o}} \mid \operatorname{db}_{j}^{\text {ref }}(\omega)\right\}\left.\right|^{2} w_{j}(\omega) d \omega \quad, \quad c_{\text {std }}=\sum_{j=1}^{m_{o}} \int_{\mathcal{B}_{o}}\left|v_{j}^{\text {ref }, n)}(\omega)\right|^{2} w_{j}(\omega) d \omega .
$$


In these equations, $\mathbf{v}^{(\mathrm{ref}, n)}(\omega)=\left(v_{1}^{(\mathrm{ref}, n)}(\omega), \ldots, v_{m_{o}}^{(\mathrm{ref}, n)}(\omega)\right)$ and $\mathbf{v}^{(n)}(\omega ; \boldsymbol{\alpha})=\left(v_{1}^{(n)}(\omega ; \boldsymbol{\alpha}), \ldots\right.$, $\left.v_{m_{o}}^{(n)}(\omega ; \boldsymbol{\alpha})\right)$ are defined, for $j=1, \ldots m_{o}$, by

$$
v_{j}^{(\mathrm{ref}, n)}(\omega)=\gamma\left|\mathrm{db}_{j}^{\mathrm{ref}}(\omega)-\mathrm{db}_{j}^{(n)}(\omega)\right|,
$$

in which $\gamma>0$ is an amplitude factor and where

$$
\left.v_{j}^{(n)}(\omega ; \boldsymbol{\alpha})=\left\{E\left\{d B_{j}^{(n)}(\omega ; \boldsymbol{\alpha})^{2}\right\}-\left(E\left\{d B_{j}^{(n)}(\omega ; \boldsymbol{\alpha})\right\}\right)^{2}\right\}\right\}^{1 / 2} .
$$

In Eqs. (77) to (79), for $j=1, \ldots m_{o}$, the functions $\omega \mapsto w_{j}(\omega)$ are bounded on $\mathcal{B}_{o}$ with values in $\mathbb{R}^{+}$and are chosen such that $w_{j}(\omega)=\left|\mathrm{db}_{j}^{\text {ref }}(\omega)-\mathrm{db}_{j}^{(n)}(\omega)\right|$ for all $\omega$ in $\mathcal{B}_{o}$. The weight (in the cost function) is $w_{J}=0.9$, and the amplitude factor (for the target) is $\gamma=0.3$. The optimization problem

$$
\boldsymbol{\alpha}^{\mathrm{opt}}=\left(s^{\mathrm{opt}}, \beta^{\mathrm{opt}},\left[\sigma^{\mathrm{opt}}\right]\right)=\min _{\boldsymbol{\alpha} \in \mathcal{C}_{\boldsymbol{\alpha}}} J(\boldsymbol{\alpha}),
$$

is solved using the algorithm detailed in [65], which is based on the use of the interior-point algorithm with constraints. The Monte Carlo solver is used with 1000 independent realizations (mean-square convergence reached). The optimal value of $s$ and $\beta$ are $s^{\text {opt }}=0.0103$ and $\beta^{\text {opt }}=0.0181$ and the optimal value $\left[\sigma^{\text {opt }}\right]$ of $[\sigma]$ can be found in [65] .

(iii) Results. In Fig. 4, the four figures that are displayed summarize the results obtained with the SROM. These four figures are related to the $x_{2^{-}}$and $x_{3}$-accelerations of $\mathrm{Obs}_{51}$ and $\mathrm{Obs}_{34}$. Each figure displays the graph $\nu \mapsto \log _{10}\left(\left|\widehat{o}_{j}(2 \pi \nu)\right|\right)$ computed with the HFM (the target for the mean), the graph $\nu \mapsto \log _{10}\left(\left|\widehat{o}_{j}^{(n)}(2 \pi \nu)\right|\right)$ computed with the linear ROM, and the confidence region (with a probability $p_{c}=0.98$ ) of the frequency sampled stochastic process $\nu \mapsto \log _{10}\left(\left|\widehat{O}_{j}^{(n)}(2 \pi \nu)\right|\right)$ constructed with the SROM. The upper envelope of the confidence region corresponds to the quantile for the probability $p_{c}$ and the lower envelope to the quantile for the probability $1-p_{c}$. It can be seen that the results obtained are very good, except for the $x_{3}$-accelerations in the small part $[270,438] \mathrm{Hz}$ of the frequency band $[0,1550] \mathrm{Hz}$ of analysis. Such a relatively bad prediction could certainly be improved using a much more sophisticated optimization algorithm for the identification of the hyperparameters. Nevertheless, it can be seen that the SROM allows for generating a confidence region, which is not centered around the responses computed with the ROM, but which is approximatively well centered around the responses computed with the HFM, which is a relatively difficult problem for taking into account contributions of second-order. Such a result demonstrates the capability of method proposed.

\section{CONCLUSIONS}

In this paper, a novel nonparametric probabilistic approach has been presented for taking into account modeling errors in any nonlinear high-fidelity model (HFM) for which a nonlinear ROM can be constructed. The nonparametric probabilistic model is implemented in the nonlinear ROM. The proposed stochastic model exhibits a small number of hyperparameters, which allows their identification by solving a statistical inverse problem. The cost function is formulated with respect to a given target related to given observations, which allows for specifying the level of uncertainties induced by the use of the ROM instead of the HFM and/or by the modeling errors (model form uncertainties) introduced in the HFM with respect to experimental data. The least-square method that is used in the paper can be replaced by another statistical inverse method such as the maximum likelihood method. A first validation of the proposed method 

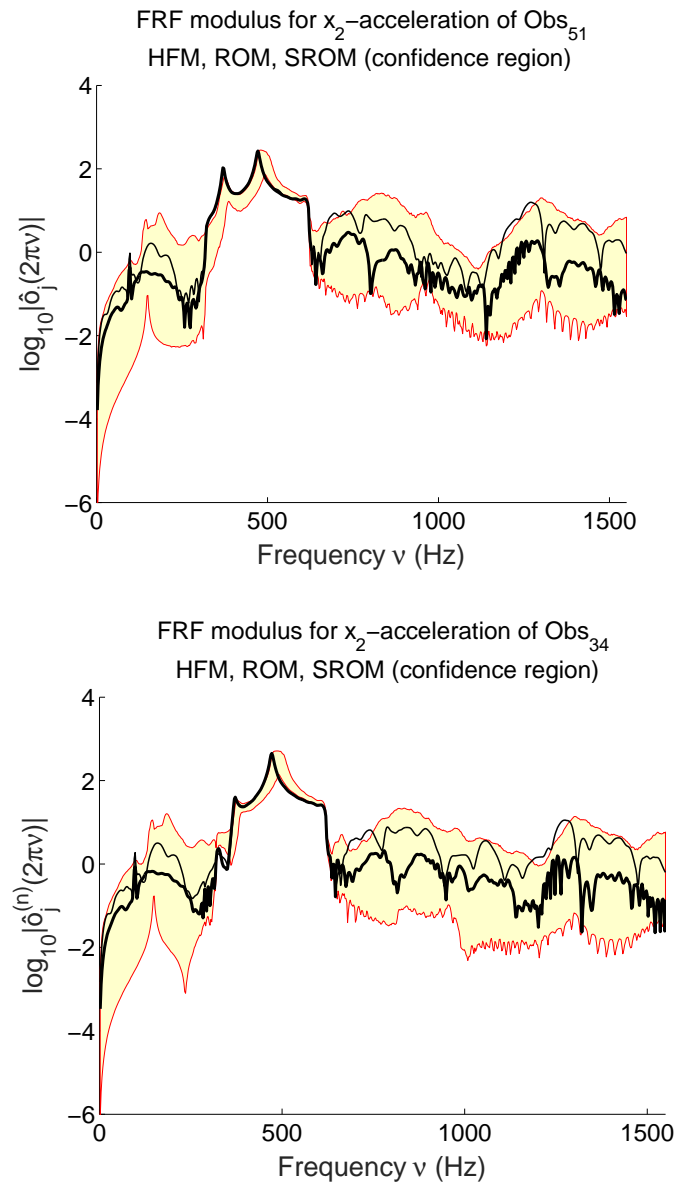
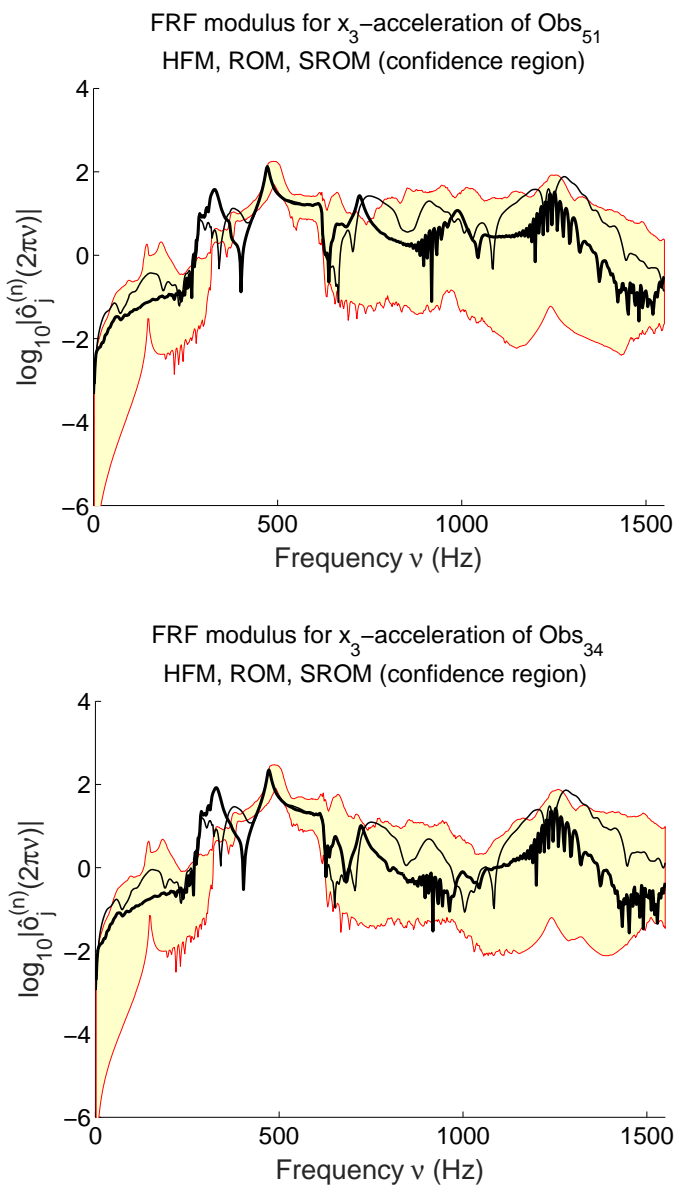

Figure 4: Graphs $\nu \mapsto \log _{10}\left(\left|\widehat{o}_{j}(2 \pi \nu)\right|\right)$ computed with the HFM (thick lines), graphs $\nu \mapsto \log _{10}\left(\left|\widehat{o}_{j}^{(n)}(2 \pi \nu)\right|\right)$ computed with the ROM (thin lines), and confidence region for $p_{c}=0.98$ (yellow region with red upper and red lower envelopes) computed for $\nu \mapsto \log _{10}\left(\left|\widehat{O}_{j}^{(n)}(2 \pi \nu)\right|\right)$ computed with the SROM, for $x_{2}$ - and $x_{3}$-accelerations of $\mathrm{Obs}_{51}$ (up left and up right figures), and for $x_{2}$ - and $x_{3}$-accelerations of $\mathrm{Obs}_{34}$ (down left and down right figures).

has been obtained by analyzing a nonlinear dynamical problem. The proposed nonparametric probabilistic approach of modeling errors can simultaneously be used with the parametric probabilistic approach of the uncertain parameters of the computational model, because, if the construction of the ROB can depend on these random parameters, the construction of the SROB does not directly depend on them (but depends indirectly through the ROB). In addition, if the Monte Carlo method is used as stochastic solver, the realizations of the SROB and those of the random parameters are constructed simultaneously. Consequently, the speed of convergence (that does not depends on the dimension) is not altered if the parametric uncertainties are taken into account in the computational model. The method can be extended to the case of a HFM for which the level of model uncertainties is not the same in the different parts of the system.

\section{ACKNOWLEDGEMENTS}

The second author acknowledges partial support by the Army Research Laboratory through the Army High Performance Computing Research Center under Cooperative Agreement W911NF07-2-0027, partial support by The Boeing Company under Contract Sponsor Ref. 45047, and partial support by DARPA under the Enabling Quantification of Uncertainty in Physical Sys- 
tems (EQUiPS) program. This document does not necessarily reflect the position of these institutions, and no official endorsement should be inferred.

\section{REFERENCES}

[1] M.A. Grepl, Y. Maday, N.C. Nguyen, A. Patera, Efficient reduced-basis treatment of nonaffine and nonlinear partial differential equations. ESAIM: Mathematical Modelling and Numerical Analysis, 41(03), 575-605, 2007.

[2] N. Nguyen, J. Peraire, An efficient reduced-order modeling approach for non-linear parametrized partial differential equations. International Journal for Numerical Methods in Engineering, 76(1), 27-55, 2008.

[3] S. Chaturantabut, D.C. Sorensen, Nonlinear model reduction via discrete empirical interpolation. SIAM Journal on Scientific and Statistical Computing, 32(5), 2737-2764, 2010.

[4] J. Degroote, J. Virendeels, K. Willcox, Interpolation among reduced-order matrices to obtain parameterized models for design, optimization and probabilistic analysis. International Journal for Numerical Methods in Fluids, 63, 207-230, 2010.

[5] K. Carlberg, C. Bou-Mosleh, C. Farhat, Efficient non-linear model reduction via a leastsquares Petrov-Galerkin projection and compressive tensor approximations. International Journal for Numerical Methods in Engineering, 86(2), 155-181, 2011.

[6] K. Carlberg, C. Farhat, A low-cost, goal-oriented compact proper orthogonal decomposition basis for model reduction of static systems. International Journal for Numerical Methods in Engineering, 86(3), 381-402, 2011.

[7] D. Amsallem, M.J. Zahr, C. Farhat, Nonlinear model order reduction based on local reduced-order bases. International Journal for Numerical Methods in Engineering, 92(10), 891-916, 2012.

[8] K. Carlberg, C. Farhat, J. Cortial, D. Amsallem, The GNAT method for nonlinear model reduction: effective implementation and application to computational fluid dynamics and turbulent flows. Journal of Computational Physics, 242, 623-647, 2013.

[9] M. Zahr, C. Farhat, Progressive construction of a parametric reduced-order model for PDE-constrained optimization. International Journal for Numerical Methods in Engineering, 102(5), 1077-1110, 2015.

[10] D. Amsallem, M. Zahr, Y. Choi, C. Farhat, Design optimization using hyper-reduced-order models. Structural and Multidisciplinary Optimization, 51(4), 919-940, 2015.

[11] A. Paul-Dubois-Taine, D. Amsallem, An adaptive and efficient greedy procedure for the optimal training of parametric reduced-order models. International Journal for Numerical Methods in Engineering, 102(5), 1262-1292, 2015.

[12] C. Farhat, P. Avery, T. Chapman, J. Cortial, Dimensional reduction of nonlinear finite element dynamic models with finite rotations and energy-based mesh sampling and weighting for computational efficiency. International Journal for Numerical Methods in Engineering, 98(9), 625-662, 2014. 
[13] C. Farhat, T. Chapman, P. Avery, Structure-preserving, stability, and accuracy properties of the Energy-Conserving Sampling and Weighting (ECSW) method for the hyper reduction of nonlinear finite element dynamic models. International Journal for Numerical Methods in Engineering, 102(5), 1077-1110, 2015.

[14] D. Ryckelynck, A priori hyperreduction method: an adaptive approach. Journal of Computational Physics, 202, 346-366, 2005.

[15] R. Ghanem, P.D. Spanos, Stochastic Finite Elements: a Spectral Approach. SpringerVerlag, New York, 1991.

[16] R. Ghanem, P.D. Spanos, Stochastic Finite Elements: A spectral Approach (revised edition). Dover Publications, New York, 2003.

[17] C. Soize, R. Ghanem, Physical systems with random uncertainties: Chaos representation with arbitrary probability measure. SIAM Journal On Scientific Computing, 26(2), 395410, 2004.

[18] R. Mace, W. Worden, G. Manson, Uncertainty in Structural Dynamics, Special issue of the Journal of Sound and Vibration, 288(3), 431-790, 2005.

[19] G.I. Schueller, Computational Methods in Stochastic Mechanics and Reliability Analysis. Special issue of Computer Methods in Applied Mechanics and Engineering, 194(12-16), 1251-1795, 2005.

[20] G.I. Schueller, Uncertainties in Structural Mechanics and Analysis-Computational Methods. Special issue of Computer and Structures, 83(14), 1031-1150, 2005.

[21] G.I. Schueller, Developments in stochastic structural mechanics. Archive of Applied Mechanics, 75(10-12), 755-773, 2006.

[22] G. Deodatis, P.D. Spanos, 5th International Conference on Computational Stochastic Mechanics. Special issue of the Probabilistic Engineering Mechanics, 23(2-3), 103-346, 2008.

[23] G.I. Schueller, H.J. Pradlwarter, Uncertain linear systems in dynamics: Retrospective and recent developments by stochastic approaches. Engineering Structures, 31(11), 2507 $2517,2009$.

[24] O.P. Le Maitre, O.M. Knio, Spectral Methods for Uncerainty Quantification with Applications to Computational Fluid Dynamics. Springer, Heidelberg, 2010.

[25] C. Soize, Stochastic Models of Uncertainties in Computational Mechanics. American Society of Civil Engineers (ASCE), Reston, 2012.

[26] T. Bui-Thanh, K. Willcox, O. Ghattas, Parametric reduced-order models for probabilistic analysis of unsteady aerodynamic applications. AIAA Journal, 46(10), 2520-2529, 2008.

[27] C. Soize, Stochastic modeling of uncertainties in computational structural dynamics - Recent theoretical advances. Journal of Sound and Vibration, 332(10), 2379-2395, 2013. 
[28] Y.M. Marzouk, H.N. Najm, L.A. Rahn, Stochastic spectral methods for efficient Bayesian solution of inverse problems. Journal of Computational Physics, 224(2), 560-586, 2007.

[29] D. Galbally, K. Fidkowski, K. Willcox, O. Ghattas, Non-linear model reduction for uncertainty quantification in large scale inverse problems. International Journal for Numerical Methods in Engineering, 81(12), 1581-1608, 2010.

[30] C. Lieberman, K. Willcox, O. Ghattas, Parameter and state model reduction for large scale statistical inverse problems. SIAM Journal on Scientific Computing, 32, 2523-2542, 2010.

[31] C. Soize, Identification of high-dimension polynomial chaos expansions with random coefficients for non-Gaussian tensor-valued random fields using partial and limited experimental data. Computer Methods in Applied Mechanics and Engineering, 199(33-36), 2150-2164, 2010.

[32] C. Soize, A computational inverse method for identification of non-Gaussian random fields using the Bayesian approach in very high dimension. Computer Methods in Applied Mechanics and Engineering, 200(45-46), 3083-3099, 2011.

[33] A. Nouy, C. Soize, Random fields representations for stochastic elliptic boundary value problems and statistical inverse problems. European Journal of Applied Mathematics, 25(3), 339-373, 2014.

[34] T. Cui, Y.M. Marzouk, K. Willcox, Data-driven model reduction for the Bayesian solution of inverse problems. International Journal for Numerical Methods in Engineering, 102(5), 966-990, 2015.

[35] C. Soize, Random vectors and random fields in high dimension. Parametric model-based representation, identification from data, and inverse problems. pp:1-65, in Handbook for Uncertainty Quantification, edited by Ghanem R, Higdon D, Owhadi H., Springer, Heidelberg, 2016.

[36] C. Soize A nonparametric model of random uncertainties for reduced matrix models in structural dynamics. Probabilistic Engineering Mechanics, 15(3), 277-294, 2000.

[37] C. Soize, Maximum entropy approach for modeling random uncertainties in transient elastodynamics. Journal of the Acoustical Society of America, 109(5), 1979-1996, 2001.

[38] C. Soize, Random matrix theory for modeling random uncertainties in computational mechanics. Computer Methods in Applied Mechanics and Engineering, 194(12-16), 13331366, 2005.

[39] C. Soize, Random Matrix Models and Nonparametric Method for Uncertainty Quantification. pp:1-84, Handbook for Uncertainty Quantification, edited by Ghanem R, Higdon D, Owhadi H., Springer, Heidelberg, 2016.

[40] C.E. Shannon, A mathematical theory of communication. Bell System Technical Journal, 27,379-423 and 623-659, 1948.

[41] E.T. Jaynes, Information theory and statistical mechanics. Physical Review, 106(4), 620630 and 108(2), 171-190, 1957. 
[42] J.N. Kapur, H.K. Kesavan, Entropy Optimization Principles with Applications. Academic Press, San Diego, 1992.

[43] C. Soize, Construction of probability distributions in high dimension using the maximum entropy principle. Applications to stochastic processes, random fields and random matrices. International Journal for Numerical Methods in Engineering, 76(10), 1583-1611, 2008.

[44] A. Batou, C. Soize, Calculation of Lagrange multipliers in the construction of maximum entropy distributions in high stochastic dimension. SIAM/ASA Journal on Uncertainty Quantification, 1(1),431-451, 2013.

[45] M.P. Mignolet, C. Soize, Nonparametric stochastic modeling of linear systems with prescribed variance of several natural frequencies. Probabilistic Engineering Mechanics, 23(2-3), 267-278, 2008.

[46] C. Soize, Nonparametric probabilistic approach of uncertainties for elliptic boundary value problem. International Journal for Numerical Methods in Engineering, 80(6-7), 673-688, 2009.

[47] C. Chen, D. Duhamel, C. Soize, Probabilistic approach for model and data uncertainties and its experimental identification in structural dynamics: Case of composite sandwich panels. Journal of Sound and Vibration, 294(1-2),64-81, 2006.

[48] R. Ohayon, C. Soize, Advanced Computational Vibroacoustics. Reduced-Order Models and Uncertainty Quantification. Cambridge University Press, Cambridge, 2014.

[49] C. Soize, I.E. Poloskov, Time-domain formulation in computational dynamics for linear viscoelastic media with model uncertainties and stochastic excitation. Computers and Mathematics with Applications, 64(11), 3594-3612, 2012.

[50] C. Soize, H. Chebli, Random uncertainties model in dynamic substructuring using a nonparametric probabilistic model. Journal of Engineering Mechanics, 129(4),449-457, 2003.

[51] H. Chebli, C. Soize, Experimental validation of a nonparametric probabilistic model of non homogeneous uncertainties for dynamical systems. Journal of the Acoustical Society of America, 115(2), 697-705, 2004.

[52] E. Capiez-Lernout, C. Soize, J.P. Lombard, C. Dupont, E. Seinturier, Blade manufacturing tolerances definition for a mistuned industrial bladed disk. Journal of Engineering for Gas Turbines and Power, 127(3), 621-628, 2005.

[53] J. Duchereau, C. Soize, Transient dynamics in structures with nonhomogeneous uncertainties induced by complex joints. Mechanical Systems and Signal Processing, 20(4), 854-867, 2006.

[54] M.P. Mignolet, C. Soize, J. Avalos, Nonparametric stochastic modeling of structures with uncertain boundary conditions / coupling between substructures. AIAA Journal, 51(6), 1296-1308, 2013. 
[55] J.F. Durand, C. Soize, L. Gagliardini, Structural-acoustic modeling of automotive vehicles in presence of uncertainties and experimental identification and validation. Journal of the Acoustical Society of America, 124(3), 1513-1525, 2008.

[56] C. Fernandez, C. Soize, L. Gagliardini, Sound-insulation layer modelling in car computational vibroacoustics in the medium-frequency range. Acta Acustica United with Acustica (AAUWA), 96(3), 437-444, 2010.

[57] R. Ohayon, C. Soize, Advanced computational dissipative structural acoustics and fluidstructure interaction in low- and medium-frequency domains. Reduced-order models and uncertainty quantification. International Journal of Aeronautical and Space Sciences, 13(2), 127-153, 2012.

[58] C. Desceliers, C. Soize, S. Cambier, Non-parametric-parametric model for random uncertainties in nonlinear structural dynamics - Application to earthquake engineering. Earthquake Engineering and Structural Dynamics, 33(3), 315-327, 2004.

[59] R. Cottereau, D. Clouteau, C. Soize, Construction of a probabilistic model for impedance matrices. Computer Methods in Applied Mechanics and Engineering, 196(17-20), 22522268, 2007.

[60] R. Cottereau, D. Clouteau, C. Soize, Probabilistic impedance of foundation: impact of the seismic design on uncertain soils. Earthquake Engineering and Structural Dynamics, 37(6), 899-918, 2008.

[61] E. Capiez-Lernout, C. Soize, Design optimization with an uncertain vibroacoustic model. Journal of Vibration and Acoustics, 130(2), 1-8, 2008.

[62] E. Capiez-Lernout, C. Soize, Robust design optimization in computational mechanics. Journal of Applied Mechanics - Transactions of the ASME, 75(2),1-11, 2008.

[63] M.P. Mignolet, C. Soize, Stochastic reduced order models for uncertain nonlinear dynamical systems. Computer Methods in Applied Mechanics and Engineering, 197(45-48), 3951-3963, 2008.

[64] E. Capiez-Lernout, C. Soize, M.P. Mignolet, Post-buckling nonlinear static and dynamical analyses of uncertain cylindrical shells and experimental validation. Computer Methods in Applied Mechanics and Engineering, 271(1), 210-230, 2014.

[65] C. Soize, C. Farhat, A nonparametric probabilistic approach for quantifying uncertainties in low- and high-dimensional nonlinear models. International Journal for Numerical Methods in Engineering, submitted 1st February, 2016.

[66] A. Edelman, T.A. Arias, S.T. Smith, The geometry of algorithms with orthogonality constraints. SIAM Journal on Matrix Analysis and Applications, 20(2), 303-353, 1998.

[67] P.A. Absil, R. Mahony, R. Sepulchre, Optimization Algorithms on Matrix Manifolds. Princeton University Press, Princeton, 2008.

[68] G.H. Golub, C.F. Van Loan, Matrix Computations (Third Edition). The Johns Hopkins University Press, Baltimore, 1996. 
[69] K.J. Bathe, E.L. Wilson, Numerical Methods in Finite Element Analysis. Prentice-Hall, Inc., Englewood Cliffs, New Jersey, 1976. 